OPEN ACCESS

Edited by:

Roger Chammas,

University of São Paulo, Brazi

Reviewed by:

Clarissa Ribeiro Reily Rocha

Federal University of São Paulo, Brazil

Évila Salles,

Augusta University, United States

${ }^{*}$ Correspondence:

Yi Huang

yihuang828@foxmail.com;

yihuang@hospital.cqmu.edu.cn

Specialty section:

This article was submitted to

Molecular and Cellular Oncology,

a section of the journal

Frontiers in Oncology

Received: 12 March 2021

Accepted: 04 May 2021

Published: 26 May 2021

Citation:

Li R, Zeng $X$, Yang $M, X u X$, Feng J,

Bao L, Xue B, Wang $X$ and Huang $Y$

(2021) Antidiabetic Agent DPP-4i

Facilitates Murine Breast Cancer Metastasis by Oncogenic ROS-NRF2HO-1 Axis via a Positive NRF2-HO-1

Feedback Loop.

Front. Oncol. 11:679816.

doi: 10.3389/fonc.2021.679816

\section{Antidiabetic Agent DPP-4i Facilitates Murine Breast Cancer Metastasis by Oncogenic ROS-NRF2-HO-1 Axis via a Positive NRF2-HO-1 Feedback Loop}

\author{
Rui $L i^{1}$, Xin Zeng ${ }^{1}$, Meihua Yang ${ }^{2}$, Xiaohui $X u^{1}$, Jinmei Feng ${ }^{1}$, Liming Bao ${ }^{3}$, \\ Bingqian Xue ${ }^{1}$, Xin Wang ${ }^{1,4}$ and Yi Huang ${ }^{1 *}$
}

${ }^{1}$ Chongqing Key Laboratory of Child Infection and Immunity, Ministry of Education Key Laboratory of Child Development and Disorders, National Clinical Research Center for Child Health and Disorders, China International Science and Technology Cooperation Base of Child Development and Critical Disorders, Children's Hospital of Chongqing Medical University,

Chongqing, China, ${ }^{2}$ Department of Neurosurgery, Xinqiao Hospital of Third Military Medical University, Chongqing, China, ${ }^{3}$ Department of Pathology, School of Medicine, University of Colorado Anschutz Medical Campus, Aurora,

CO, United States, ${ }^{4}$ Department of Laboratory Medicine, The People's Hospital of Guangxi Zhuang Autonomous Region, Nanning, China

Cancer has been as one of common comorbidities of diabetes. Long-term antidiabetic treatment may potentially exert uncertain impacts on diabetic patients with cancer including breast cancer (BC). Dipeptidyl peptidase-4 inhibitors (DPP-4i) are currently recommended by the AACE as first-line hypoglycemic drugs in type 2 diabetes mellitus (T2DM). Although the safety of DPP-4i has been widely evaluated, the potential sideeffects of DPP-4i in cancer metastasis were also reported and remain controversial. Here, we revealed that Saxagliptin (Sax) and Sitagliptin (Sit), two common DPP-4i compounds, potentially promoted murine BC 4T1 metastasis in vitro and in vivo under immunedeficient status. Mechanically, we observed that DPP-4i treatment induced aberrant oxidative stress by triggering ROS overproduction, as well as ROS-dependent NRF2 and $\mathrm{HO}-1$ activations in $\mathrm{BC}$ cells, while specific inhibition of ROS, NRF2 or $\mathrm{HO}-1$ activations abrogated DPP-4i-driven BC metastasis and metastasis-associated gene expression in vitro. Furthermore, ALA, a NRF2 activator significantly promoted BC metastasis in vitro and in vivo, which can be abrogated by specific $\mathrm{HO}-1$ inhibition in vitro. Moreover, specific HO-1 inhibition not only reversed DPP-4i-induced NRF2 activation but also abrogated ALA-induced NRF2 activation, resulting in a decrease of metastasis-associated genes, indicating a positive-feedback NRF2-HO-1 loop. Our findings suggest that DPP-4i accelerates murine $\mathrm{BC}$ metastasis through an oncogenic ROS-NRF2-HO-1 axis via a positive-feedback NRF2-HO-1 loop. Therefore, this study not only offers novel insights into an oncogenic role of DPP-4i in BC progression but also provides new strategies to alleviate the dark side of DPP-4i by targeting $\mathrm{HO}-1$.

Keywords: DPP-4 inhibitor, NRF2, HO-1 (heme oxygenase-1), metastasis, breast cancer 


\section{INTRODUCTION}

Accumulating evidences suggest that diabetes increases incidence of human cancers including breast cancer (BC) (1). Long-term treatment of antidiabetic drugs may potentially have uncertain impacts on comorbid $\mathrm{BC}$ in diabetic patients. Therefore, improved understanding of the effects of antidiabetic agents in BC cells should open new avenues for minimizing the risk of antidiabetic agents in diabetic patients with BC (2). Dipeptidyl peptidase-4 inhibitors (DPP-4i), such as saxagliptin (Sax) and sitagliptin (Sit), are currently recommended by the American Association of Clinical Endocrinologists (AACE) as first-line hypoglycemic treatment in type 2 diabetes mellitus (T2DM). Although the safety of DPP4i has been widely evaluated, the potential side effects of DPP-4i in cancer metastasis were also reported and remains controversial $(3,4)$. A previous report suggested a potential antitumor role of DPP-4 inhibition in improvement of tumor immunity by regulating CXCL10-mediated lymphocyte trafficking in mice (5), whereas our recent finding revealed an oncogenic role of DPP-4i in human cancers including BC through NRF2-mediated anti-oxidative stress (6). Thus, the potential side-effects of antidiabetic agents DPP-4i in BC metastasis should be further clarified.

The cancer immunoediting concept has provided a critical insight into the crosstalk between tumor cells and immune system during the cancer development (7-11). Although there is no doubt that immunity is critical to tumor progression, tumor cells as one of the major components of tumor microenvironment, was shown to exert major contribution to tumor progression by remodeling the tumor microenvironment $(7-11)$. Thus, better understanding the effect of antidiabetic DPP-4i on existing tumors and the underlying mechanism not only would offer novel insights into its potential role in tumor progression but also may provide new strategies to alleviate the dark side of DPP-4i in diabetic patients with cancer.

Here, we utilized a murine $\mathrm{BC}$ cell line $4 \mathrm{~T} 1$, that is well known to mimic the metastatic and advanced stages of human BC (11), to investigate the effect of Sax and Sit on BC metastasis in vitro and in vivo. Then, we further investigated the possible mechanism underlying how DPP-4i regulates BC metastasis.

\section{MATERIALS AND METHODS}

\section{Cell Lines, Cell Culture, and Reagents}

Murine BC cell line 4T1 was obtained from the American Tissue Culture Collection (ATCC) as our previous reports (11). Cells were cultured in RPMI 1640 (Gibco BRL, Rockville, MD, USA) supplemented with $10 \%$ fetal bovine serum, $100 \mathrm{U} / \mathrm{ml}$ penicillin, and $100 \mu \mathrm{g} / \mathrm{ml}$ streptomycin at $37^{\circ} \mathrm{C}$ in a humidified chamber containing $5 \% \mathrm{CO}_{2}$. Neh2-Luciferase reporter vectors for NRF2/ ARE activation were reserved by our lab as described previously (12). Saxagliptin (Sax), Sitagliptin (Sit), and Heme oxygenase 1 (HO-1) specific inhibitor HO-1-IN-1 hydrochloride (10 mM in DMSO) were purchased from MedChemExpress (MCE)
(Houston, TX, USA). ROS scavenger N-acetylcysteine (NAC) and NRF2 specific inhibitor ML-385 were purchased from AbMole Bioscience (Houston, TX, USA). Alpha-lipoic acid (ALA, a NRF2 activator) was commercially obtained from Dandong Yichuang Co, China as previous describes (12). All chemical reagents were purchased from Sigma-Aldrich (St Louis, MO, USA) unless otherwise indicated.

\section{Cell Migration and Cell Invasion Assays}

Cell migration and cell invasion assays were performed in 24well non-coated or Matrigel-coated Transwell chambers $(8-\mu \mathrm{m}$ pore size, Corning, NY, USA) as described previously (12-14). Briefly, $4.5 \times 10^{4}$ cells for cell migration or $1 \times 10^{5}$ cells for cell invasion were plated in the upper chamber with $200 \mu \mathrm{l}$ of serumfree medium, and $800 \mu \mathrm{l}$ medium supplemented with $10 \%$ FBS was used as a chemoattractant in the bottom chamber. $4 \mathrm{~T} 1$ cells were treated with $\operatorname{Sax}(0,0.2,0.4 \mu \mathrm{M})$, Sit $(0,0.6,1.2 \mu \mathrm{M})$ or ALA $(0,40,60 \mu \mathrm{M})$ for 24 hours, and then were fixed and stained with Crystal Violet Staining Solution (Beyotime, Haimen, China). For pharmacological intervention assays, 4T1 cells were co-treated with Sax $(0.4 \mu \mathrm{M})$ or Sit $(1.2 \mu \mathrm{M})$, and NAC $(0,2.5,5 \mathrm{mM})$, or ML-385 $(0,5,10 \mu \mathrm{M})$, or HO-1 inhibitor $(0,5,10 \mu \mathrm{M})$, respectively. For HO-1 blockage assay, cells were co-treated with ALA $(60 \mu \mathrm{M})$ and HO-1 inhibitor $(0,5,10 \mu \mathrm{M})$, and cell migration and cell migration assays were performed as above mentioned. The images of the migrated or invaded cells were captured and cell number was counted in 5 to 10 random fields for each group and summarized as mean \pm standard deviation (SD) for statistical analysis.

\section{Spontaneous Breast Cancer Metastasis Mouse Model}

NOD-SCID mice (6-8 weeks, Female, SPF degree, $22 \pm 3$ g) were purchased from Beijing HFK Bioscience Co (Beijing, China). All mice were housed and maintained under specific pathogen-free (SPF) conditions as our described previously $(12,13)$. All procedures were approved by the Institutional Animal Care and Use Committee of Children's Hospital of of Chongqing Medical University. A spontaneous BC metastasis mouse model was established as previously described (11). Briefly, 4T1 cells $\left(1 \times 10^{5}\right)$ in $100 \mu \mathrm{l}$ PBS buffer were subcutaneously injected into in the left mammary fat pad of NOD-SCID mice. After 3 to 5 days, 4T1-bearing mice were randomly divided into two groups to receive $0.9 \% \mathrm{NaCl}$ or $15 \mathrm{mg} / \mathrm{kg}$ Sax orally daily ( $\mathrm{n}=3-5$ mice/ group). For ALA intervention in vivo, 4T1-bearing mice were randomly divided into two groups to receive intraperitoneal (i.p.) administration of $0.9 \% \mathrm{NaCl}$ or ALA $(80 \mathrm{mg} / \mathrm{kg}$ in $0.9 \% \mathrm{NaCl}$ ) three times per week ( $\mathrm{n}=3-5 \mathrm{mice} / \mathrm{group}$ ). At the end of experiments, experimental mice were sacrificed, and liver and lung tissues were harvested for the analysis of $\mathrm{H} \& \mathrm{E}$ and immunohistochemistry staining.

\section{Reactive Oxygen Species (ROS) Measurement}

Intracellular ROS and mitochondrial ROS (mROS) were measured by flow cytometry according to procedures as 
described previously $(6,12)$. Indicated cells were treated with $10 \mu \mathrm{M}$ DHE (Dihydroethidium) (Sigma-Aldrich) for $30 \mathrm{~min}$ at $37^{\circ} \mathrm{C}$. After washing with PBS, cells were resuspended in ice-cold PBS for flow cytometry analysis. mROS was measured using 5.0 $\mu \mathrm{M}$ MitoSoX Red probe (Thermo Fisher Scientific) according to the manufacturer's instructions. All stained cells were analyzed on a FACS Calibur flow cytometer (BD Bioscience) and data analyzed with FlowJo software (Tree Star, Ashland, OR).

\section{Intracellular Adenosine Triphosphate Level Assay}

Intracellular adenosine triphosphate (ATP) levels were measured by ATP Assay Kit (Beyotime, Haimen, China) in accordance with the manufacturer's instructions as described previously $(6,12)$. Briefly, cells in a six-well were treated with or without Sax or Sit for $24 \mathrm{~h}$ and homogenized with ice-cold lysis buffer. After centrifuged for $5 \mathrm{~min}$ at $12,000 \mathrm{~g}, 4^{\circ} \mathrm{C}$, the supernatant was used for RLU value detection using a Synergy H1 microplate reader (Bio Tek).

\section{Intracellular Reduced Glutathione/ Oxidized Glutathione ratio and NADP+/ NADPH Ratio Analysis}

The NADP+/NADPH ratio and reduced glutathione (GSH)/ oxidized glutathione (GSSG) ratio were determined using the $\mathrm{NADP}+\mathrm{NADPH}$ Assay Kit (Beyotime, Haimen, China) and GSH/GSSG Ratio Detection Assay Kit (Beyotime, Haimen, China), respectively. These assays were performed to examine the oxidative status of the cells pretreated with or without Sax or Sit according to the manufacturer's instructions as described previously $(6,12)$.

\section{RNA Isolation and Quantitative Real-Time PCR (qRT-PCR)}

RNA isolation and qRT-PCR were performed as described previously (12-17). Total RNA from harvested cells was isolated using Tripure Isolation Reagent (Roche, Mannheim, Germany). 0.5-1.0 $\mu \mathrm{g}$ total RNA was reverse-transcribed into cDNA using the PrimeScript ${ }^{\mathrm{TM}} \mathrm{RT}$ reagent Kit with gDNA Erase r(Takara, Japan) according to the manufacturer's instructions. qRT-PCR was performed with QuantiNova SYBR Green PCR Kit (Qiagen, Germany) on CFX Connect ${ }^{\mathrm{TM}}$ Real-Time System (BIO-RAD) according to the manufacturer's instructions. The relative gene expressions were normalized to the house-keeping $\beta$-actin gene and calculated using the $2^{-\Delta \Delta \mathrm{Ct}}$ method. The details of the primers are listed in Supplementary Table S1.

\section{Western Blotting}

$4 \mathrm{~T} 1$ cells were treated with indicated regents and then subject to Western blotting analysis using RIPA buffer (Beyotime, Haimen, China) as described previously (12-16). Protein lysates after SDS-PAGE were blotted onto PVDF membranes, blocked in QuickBlock $^{\mathrm{TM}}$ Blocking Buffer for Western Blot (Beyotime, Haimen, China) and followed by primary antibody incubation. Blots were washed with TBST and detected with the ECL system. All antibodies used in this study are listed in Supplementary Table S2.

\section{Luciferase Reporter Assays}

Luciferase reporter assay was performed as our described previously $(12,13)$. Briefly, cells were seeded in 96-well plates at approximately $1 \times 10^{4}$ cells per well and then transfected with the Neh2-Luciferase reporter vectors (90 ng) using X-tremeGENE HP DNA Transfection Reagent (Roche, Germany). pRL-TK Reniila plasmids (10 ng) (Promega, Madison, WI, USA) were cotransfected to normalize transfection efficiency. After transfection 16 to $18 \mathrm{~h}$, indicated regents, such as inhibitors or activators, were added as stimulation groups. After another $24 \mathrm{~h}$ incubation, the Firefly and Renilla luciferase activities were quantified using the Dual-Glo ${ }^{\circledR}$ Luciferase Assay System (Promega, Madison, USA). The relative luciferase (Luc) activity was present as the fold-change of in Firefly luciferase activity after normalization to the Renilla luciferase activity.

\section{H\&E Staining and Immunohistochemistry (IHC)}

$\mathrm{H} \& \mathrm{E}$ and IHC staining were performed as described previously $(6,12-16)$. Briefly, lung or liver tissues were fixed with $10 \%$ buffered formalin and embedded in paraffin. Tissue sections (4 $\mu \mathrm{m})$ were subjected to $\mathrm{H} \& \mathrm{E}$ staining. IHC staining was performed using Elivision plus Polyer HRP IHC Kit (Maixin, Fujian, China) and DAB kit (ZSGB-Bio, Beijing, China) according to the manufacturer's instructions.

\section{Immunofluorescence}

Immunofluorescence (IF) was performed as described previously $(6,12,13)$. Briefly, cells were fixed with $4 \%$ paraformaldehyde for $30 \mathrm{~min}$ and blocked with QuickBlock ${ }^{\mathrm{TM}}$ Blocking Buffer (Beyotime, Haimen, China) for $15 \mathrm{~min}$ at room temperature. Then cells were incubated with primary antibodies at $4^{\circ} \mathrm{C}$ overnight, followed by incubation for $2 \mathrm{~h}$ at room temperature with AF555 or AF647-conjugated secondary antibody (Biolegend). Nuclei were counterstained with 4',6diamidino-2-phenylindole (DAPI). Images were captured using a Nikon AIR Confocal Laser Microscope (Nikon, Minato, Japan) and mean fluorescent intensity (MFI) was measured by a NIS elements AR analysis software version 5.01. All antibodies used in this study are listed in Supplementary Table S2.

\section{Statistics}

Statistical analysis was carried out with the GraphPad Prism 7.0 (GraphPad Software) as previously described $(12,13)$. All data were expressed as means \pm SD. The significance of difference between groups was determined by unpaired two-tailed Student's $t$ test or the one-way analysis of variance (ANOVA). The value of $\mathrm{p}<0.05$ was considered statistically significant.

\section{RESULTS}

\section{DPP-4i (Sax and Sit) Facilitates 4T1 BC Cells Metastasis In Vitro and In Vivo}

To understand the potential role of DPP-4i in BC progression, we investigated the effect of two DPP-4i compounds, Sax and Sit 
on BC metastasis using a murine BC cell line 4T1 cells, which was used to mimic the metastatic and advanced stages of human BC (11). Firstly, we observed that Sax and Sit markedly enhanced the abilities of $\mathrm{BC}$ cell migration and cell invasion in vitro (Figures 1A, B). Furthermore, we observed that DPP-4i treatment significantly enhanced a serial of metastasisassociated gene levels including HIF-1 $\alpha$, MMP-2, MMP-9 VEGF-A, VEGF-C, VIMENTIN, and BACH-1 (Figure 1C). These results indicate that $\mathrm{DPP}-4 \mathrm{i}$ promotes $\mathrm{BC}$ migration and invasion in vitro.

To further demonstrate the direct role of DPP-4i on tumor metastasis in vivo, we subcutaneously injected $4 \mathrm{~T} 1$ cells into the left mammary fat pad of severe combined immunodeficient NODSCID mice to establish a spontaneous metastasis mice model. We observed that DPP-4i treatment significantly enhanced lung and liver metastasis of BC cells in vivo (Figure 1D). Moreover, vimentin, a micro-metastasis marker and metastasis-associated genes MMP-2, MMP-9, and VEGF levels were markedly increased in lung and liver micro-metastasis nodes (Figures 1E, F). Thus, these data indicate that DPP-4i increases BC migration and invasion by increasing metastasis-associated gene expression, thereby facilitating metastasis in vivo.

\section{ROS-Induced Aberrant Oxidative Stress Contributes to DPP-4i-Driven BC Metastasis}

Given the role of DPP-4i in the regulation to oxidative stress, we sought to know whether oxidative stress is involved in DPP-4iinduced BC metastasis. Firstly, we observed that intracellular ROS and mROS productions were significantly enhanced in DPP-4i-treated BC cells (Figures 2A, B), indicating that DPP$4 \mathrm{i}$ induces the robust release of ROS in BC cells. Then, we also assessed the effect of DPP-4i on oxidative stress status. We observed that DPP-4i significantly impaired ATP production and decreased NADP+/NADPH ratio, but markedly increased the GSH/GSSG ratio in BC cells (Supplemental Figure S1). Meanwhile, DNA damage marker 8-OHdG levels were markedly enhanced after DPP-4i treatment (Figure 2C). These data suggest that DPP-4i triggers aberrant oxidative stress by inducing ROS over production in BC cells.

To demonstrate the essential role of ROS in DPP-4i-induced $\mathrm{BC}$ metastasis, we applied the ROS scavenger-NAC intervention to assess the effect of ROS inhibition on DPP-4i-driven BC metastasis in vitro. As shown in Figures 2D, E, NAC treatment significantly abrogated DPP-4i-driven BC cell migration and invasion with a dose-dependent manner. Furthermore, DPP-4idriven metastasis-associated gene levels were also inhibited by NAC treatment (Figures 2F, G), indicating that ROS plays an oncogenic role in DPP-4i-induced BC metastasis. Therefore, these results suggest that DPP-4i drives $\mathrm{BC}$ metastasis by triggering oxidative stress via ROS overproduction.

\section{DPP-4i Induces Aberrant NRF2 Activation by a ROS-Dependent Manner}

It has been shown that DPP-4i reduced ROS-mediated oxidative stress by NRF2 activation in some human cancer cells (6), we further investigated the correlation between ROS and NRF2 in DPP-4i-treated BC cells. We found that DPP-4i significantly increased NRF2 and p-NRF2 expression (Figure 3A) and ARE-driven NRF2 transcriptional activation (Figure 3B). Meanwhile, NRF2-responsive genes expressions were significantly upregulated upon DPP-4i treatment (Figure 3C). Then, we further observed that NRF2 and p-NRF2 expressions were also increased in lung and liver metastasis tissues after DPP-4i treatment (Figure 3D). These data suggest that DPP-4i triggers aberrant NRF2 activation.

To further clarify the relationship between ROS and NRF2 activation, we investigated whether ROS inhibition could reverse DPP-4i-driven NRF2 activation. By ROS scavenger NAC intervention, we found that NAC treatment significantly attenuated NRF2 and p-NRF2 levels (Figure 3E), as well as NRF2/ARE luciferase activation (Figure 3F) in DPP-4i-treated BC cells. Furthermore, NRF2-responsive gene levels were significantly attenuated after NAC treatment in DPP-4i-treated BC cells (Figure 3G), indicating that DPP-4i induces NRF2 activation by triggering ROS. Collectively, these data indicate that DPP-4i induces aberrant NRF2 activation via ROSdependent manner.

\section{Oncogenic NRF2 Activation in DPP-4i- Driven BC Metastasis}

To define the role of NRF2 in DPP-4i-driven BC metastasis, we used ML-385, a specific NRF2 inhibitor, to explore whether pharmaceutical NRF2 inhibition could reverse DPP-4i-driven BC metastasis. We found that ML-385 treatment significantly attenuated NRF2, p-NRF2 expression (Figure 4A) and NRF2responsive gene levels (Figure 4B) in DPP-4i-treated BC cells. Notably, DPP-4i-driven BC cell migration and invasion were significantly abrogated by ML-385 treatment with a dosedependent manner (Figures 4C, D). Furthermore, DPP-4idriven metastasis-associated gene levels were significantly attenuated after ML-385 treatment in BC cells (Figure 4E). These data indicate that NRF2 inhibition reverses DPP-4idriven $\mathrm{BC}$ metastases in vitro.

To further understand the exact role of NRF2 activation in BC metastasis, we investigated the direct effect of NRF2 activator on $\mathrm{BC}$ cell migration and invasion. Using $\alpha$-lipoic acid (ALA), a well-characterized NRF2 activator, we found that pharmacological ALA treatment significantly promoted cell migration and cell invasion with a dose-dependent manner in BC cells (Figure 5A). Meanwhile, NRF2-responsive gene levels were also significantly increased after ALA treatment (Supplementary Figure S2A), indicating that pharmacological NRF2 activation promotes cell migration and invasion in vitro.

In complementary in vivo metastatic model, ALA was intraperitoneally injected into $4 \mathrm{~T} 1$-bearing mice to demonstrate the direct effect of NRF2 activation on BC metastasis. We found that ALA treatment significantly enhanced BC lung and liver metastasis (Figure 5B) while micro-metastasis marker vimentin was markedly increased in lung and liver micro-metastasis nodes in 
A

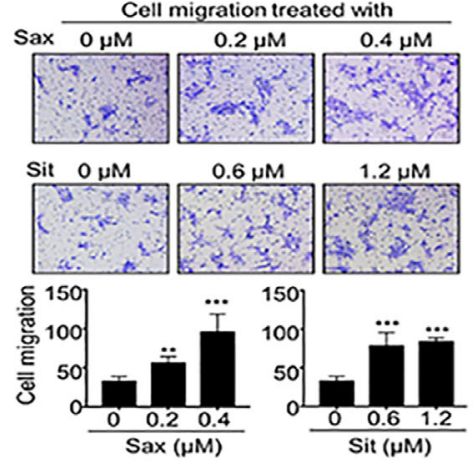

C

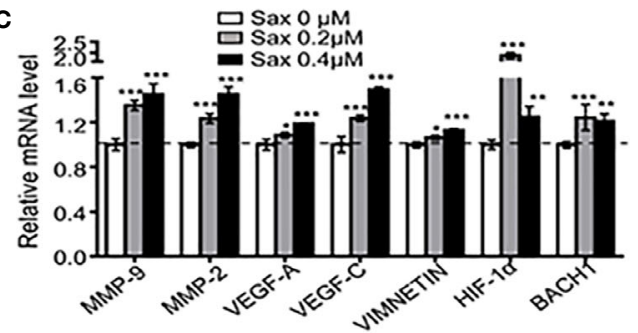

D
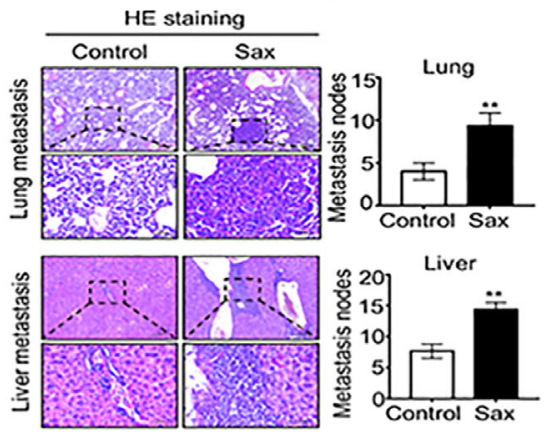

F
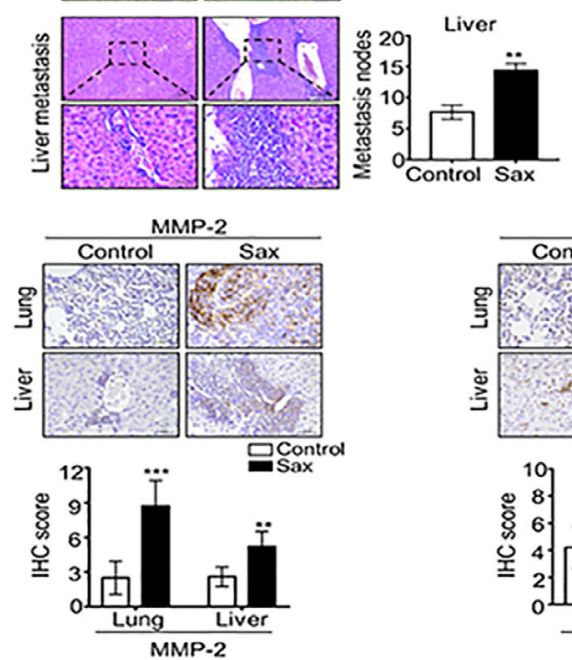
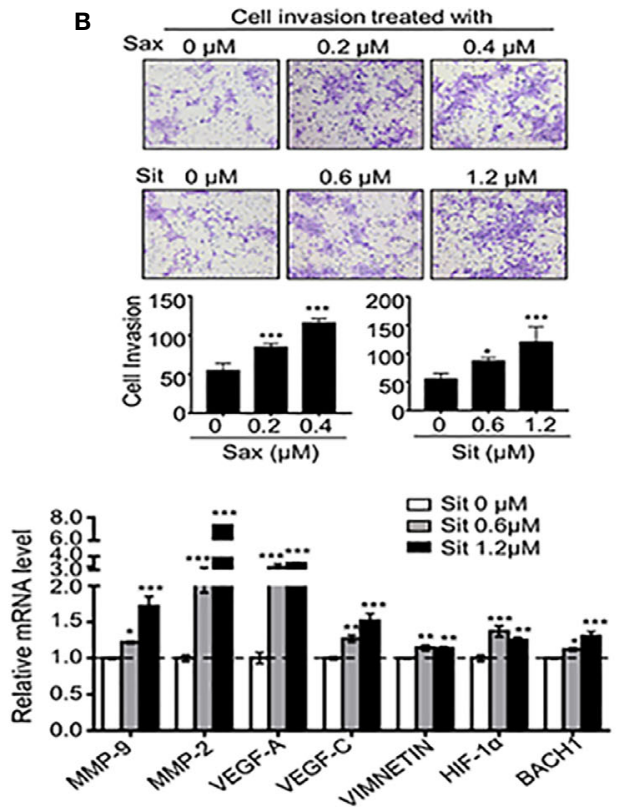

E
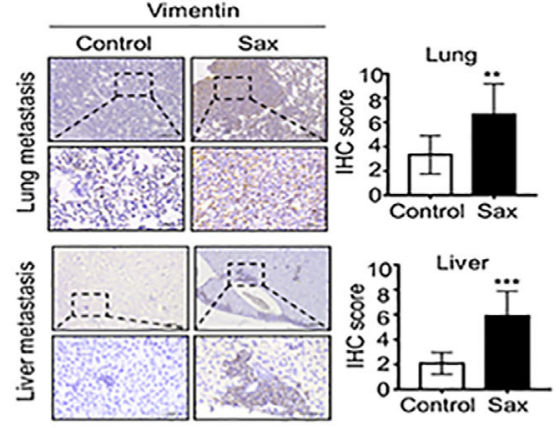
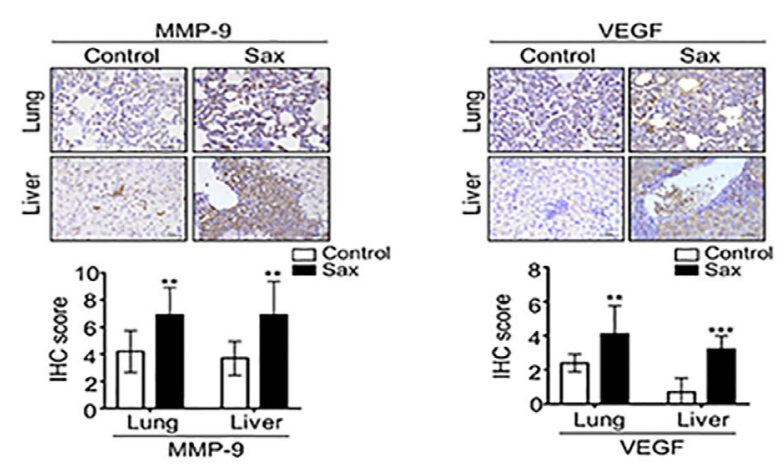

FIGURE 1 | DPP-4i(Sax and Sit)promotes murine breast cancer metastasis in vitro and in vivo. (A, B) DPP-4i (Sax and Sit) facilitates cells migration and invasion in vitro. 4T1 cells were subject to cell migration assay (A) and cell invasion analysis (B) upon treatment of Saxagliptin (Sax) or Sitagliptin (Sit). Migration or invasion cells were counted in 5 to 10 random fields. (C) DPP-4i increases metastasis-associated gene levels. 4T1 cells were co-treated with or without DPP-4i and metastasisassociated gene levels were detected by GRT-PCR. $\beta$-actin gene was as an internal control. (D) DPP-4i accelerates BC lung and liver metastasis in vivo. $1.0 \times 105$ $4 \mathrm{~T} 1$ cells in $100 \mu \mathrm{l}$ PBS buffer were injected into in the left mammary fat pad of female NOD-SCID mice. Post-injection 3 to 5 days, mice were randomly allocated to the indicated groups ( $\mathrm{n}=3-5$ mice/group) and treated with $0.9 \% \mathrm{NaCl}$ (control) or $15 \mathrm{mg} / \mathrm{kg}$ Sax via oral gavage daily. After the indicated time, lungs and livers were collected, and metastatic nodes were counted by H\&E staining (D). Micro-metastasis marker vimentin was detected by IHC staining (E). DPP-4i enhances metastasis-associated gene expression in vivo (F). 4T1-bearing mice were treated with DPP-4i (Sax) as above mentioned and metastasis-associated proteins MMP9, MMP-2 and VEGF were detected by IHC staining in lung and liver metastatic tissues. Data are presented as mean \pm SD of three independent experiments. Representative images are shown. Scale bar: $50 \mu \mathrm{m}$. ${ }^{\star} \mathrm{p}<0.05,{ }^{* \star} \mathrm{p}<0.01$, and ${ }^{\star \star *} \mathrm{p}<0.001$ between the indicated groups determined by unpaired Student's $\mathrm{t}$ test or the one-way analysis of variance (ANOVA). 


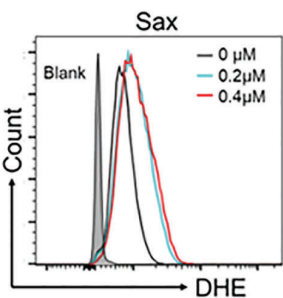

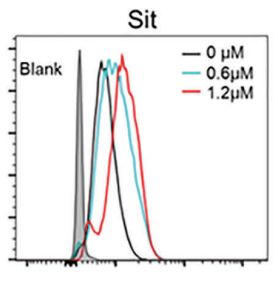

C
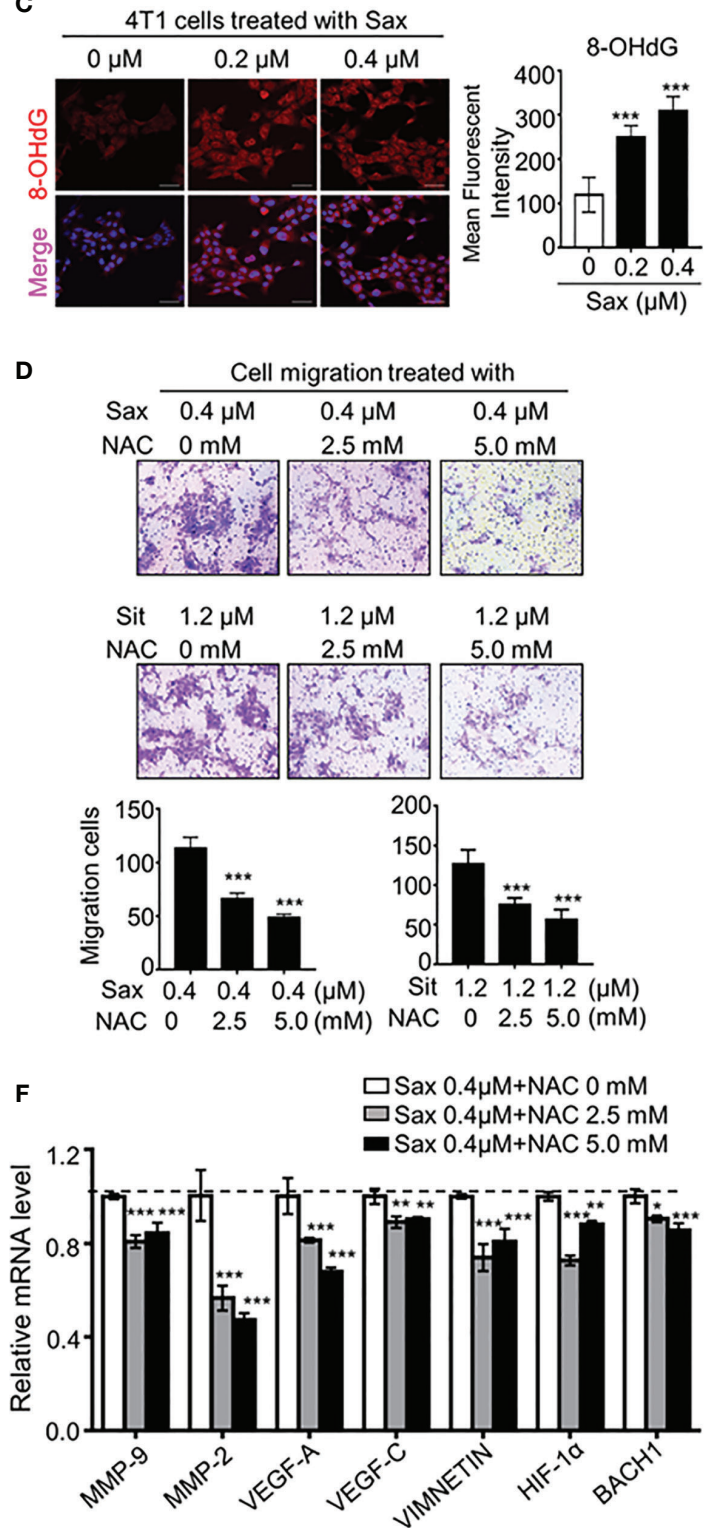
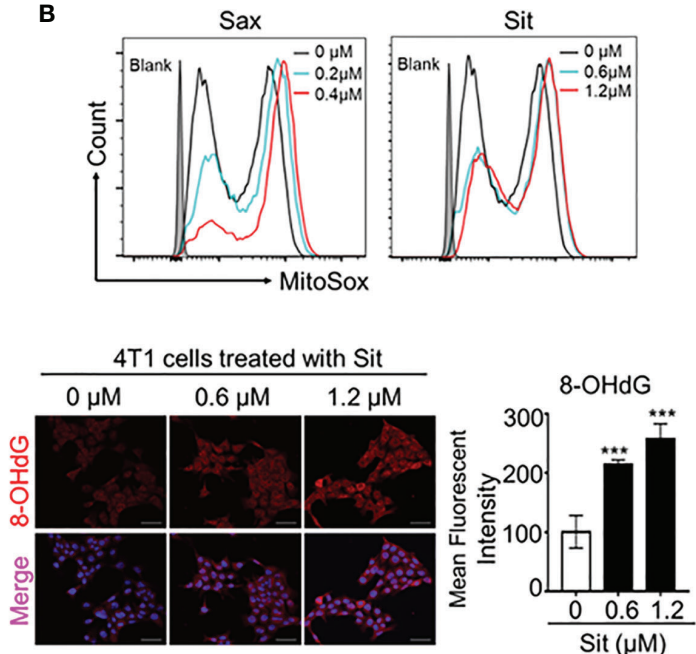

E

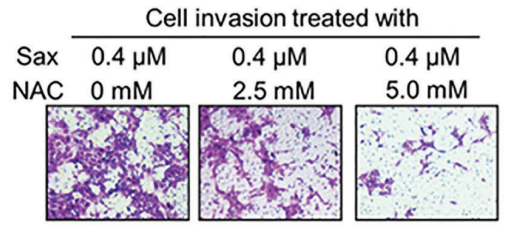

Sit $1.2 \mu \mathrm{M}$

$1.2 \mu \mathrm{M}$

$1.2 \mu \mathrm{M}$

NAC $0 \mathrm{mM}$

$2.5 \mathrm{mM}$
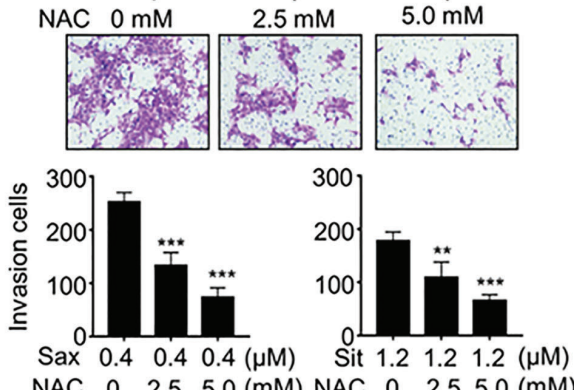

NAC $0.255 .0(\mathrm{mM})$ NAC $0 \quad 2.55 .0(\mathrm{mM})$

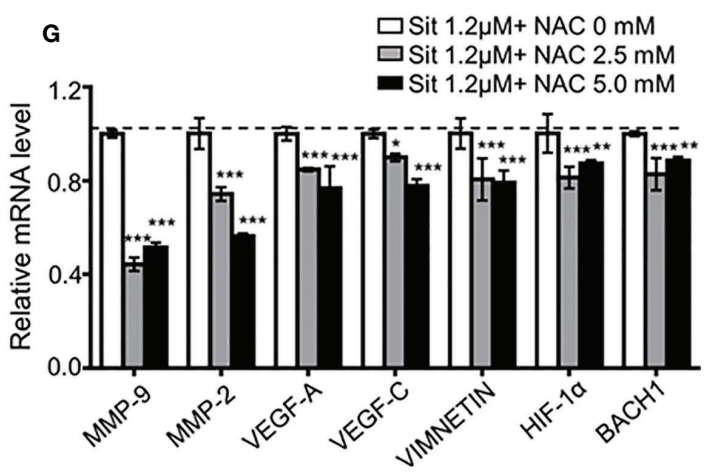

FIGURE 2 | ROS scavenger reverses DPP-4i-driven metastasis. (A, B) DPP4i triggers ROS overproduction. 4T1 cells were treated with DPP-4i. Intracellular ROS and mROS were detected in DPP-4i-treated BC cells by FCM using DHE staining (A) and MitoSox staining (B). (C) DPP-4i induces DNA damage in BC cells. 4T1 cells were treated with DPP-4i and DNA damage marker 8-oxo-deoxyguanosine (8-OHdG) was detected by IF staining. (D, E) ROS scavenger abrogates DPP-4i -driven cell migration and invasion in vitro. 4T1 cells were subject to cell migration (D) and cell invasion (E) assays upon co-treatment of DPP-4i and NAC for $24 \mathrm{~h}$. Migration or invasion cells were counted in 5 to 10 random fields. (F, G) ROS scavenger abrogates DPP-4i-driven metastasis-associated gene levels. 4T1 cells were co-treated with or without DPP-4i and NAC for $4 \mathrm{~h}$ and metastasis-associated gene levels were detected by qRT-PCR. $\beta$-actin gene was as an internal control. Data are presented as mean \pm SD of three independent experiments. Representative images are shown. Scale bars: $50 \mu m$. ${ }^{*} p<0.05,{ }^{* *} p<0.01$, and ${ }^{{ }^{* *}} p<0.001$ between the indicated groups determined by one-way analysis of variance (ANOVA). 
A

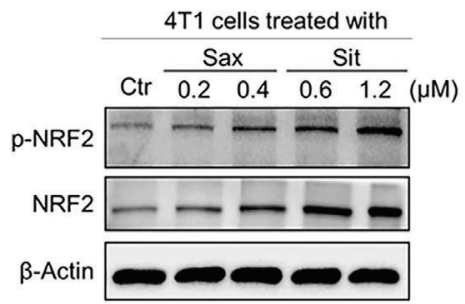

B

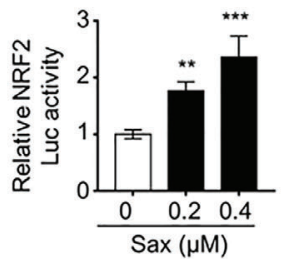

D

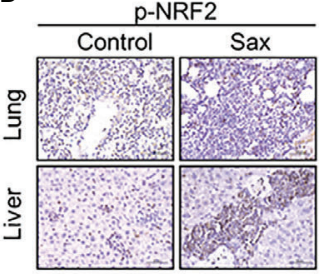

E

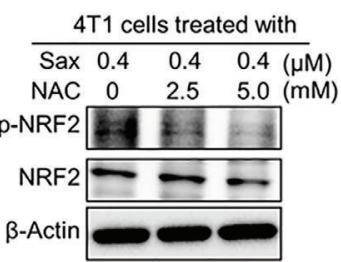

F

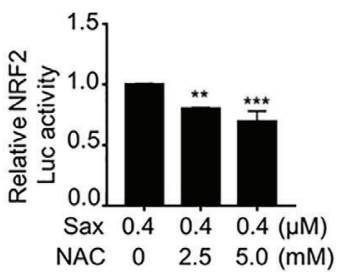

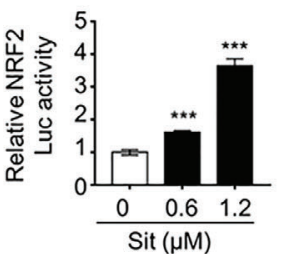
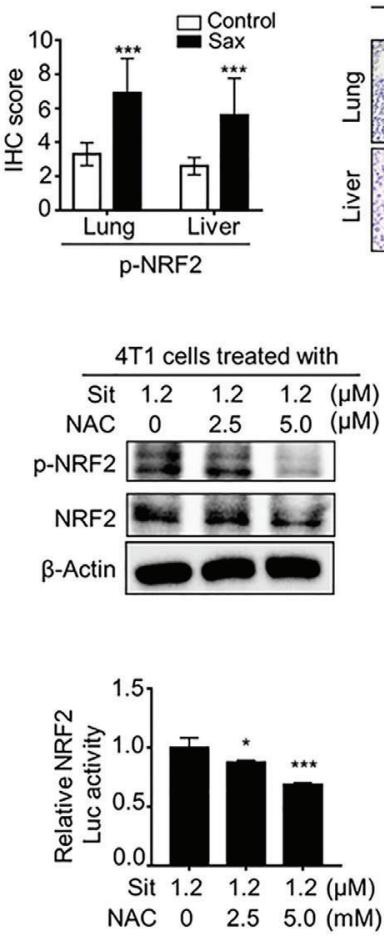
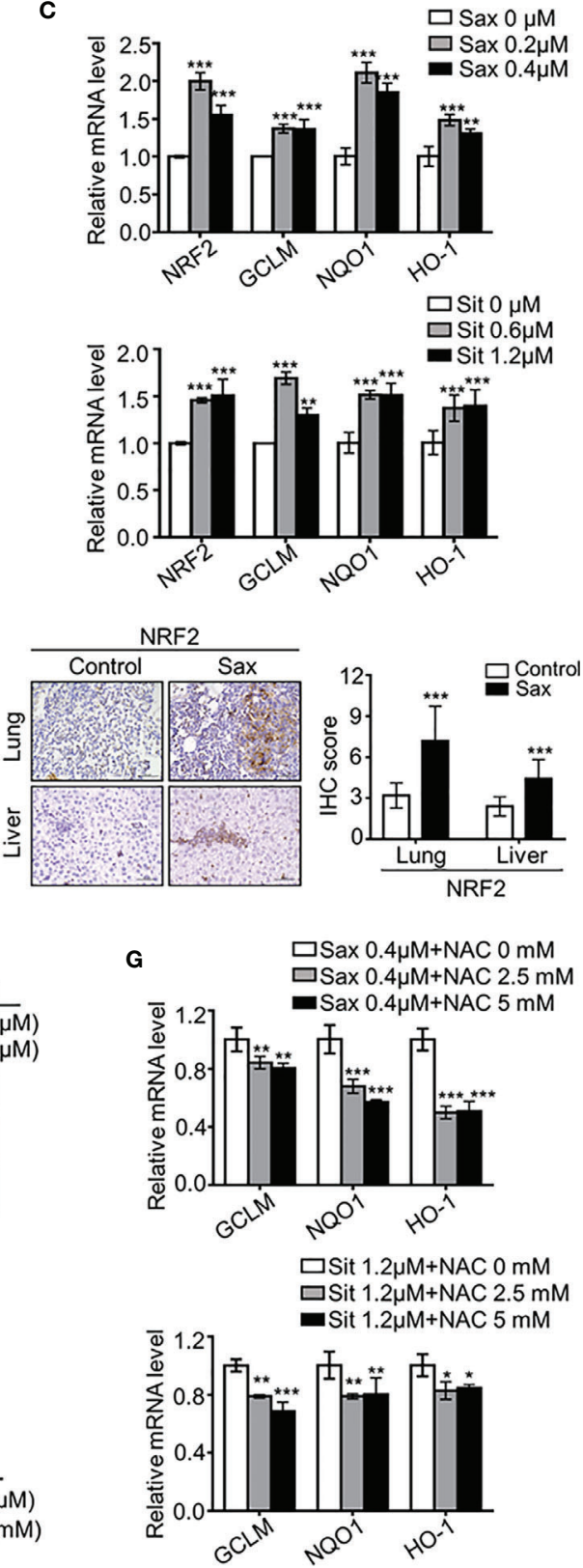

FIGURE 3 | DPP-4i induces ROS-dependent NRF2 activation. (A-C) DPP-4i enhances aberrant NRF2 activation of 4T1 cells in vitro. 4T1 cells were treated with DPP-4i. NRF2 and p-NRF2 expressions were detected by Western blotting (A). NRF2/ARE transcriptional activation was analyzed by luciferase reporter gene assay (B) and NRF2-responsive genes levels were analyzed by Real-time PCR (C). $\beta$-actin gene was as an internal control. (D) DPP-4i induces aberrant NRF2 activation in vivo. p-NRF2 and NRF2 proteins were detected by IHC staining in metastatic lung and liver tissues. (E-G) ROS scavenger abrogates DPP-4i-driven NRF2 activation. 4T1 cells were co-treated with DPP-4i and NAC. NRF2 and p-NRF2 expressions were detected by western blotting (E). NRF2/ARE transcriptional activation was analyzed by luciferase reporter gene assay (F) and NRF2-responsive genes levels were analyzed by real-time PCR (G). $\beta$-actin gene was as an internal control. Representative images are shown. Scale bars: $50 \mu \mathrm{m}$. Data are presented as mean \pm SD of three independent experiments. ${ }^{*} \mathrm{p}<0.05$, ${ }^{* *} \mathrm{p}<0.01$, and ${ }^{* \star *} \mathrm{p}<0.001$ between the indicated groups determined by unpaired Student's $t$ test or the one-way analysis of variance (ANOVA).

4T1-bearing mice (Figure 5C). Then, we also observed that ALA treatment not only enhanced NRF2 and p-NRF2 levels (Figure 5D) but also increased metastasis-associated proteins including MMP-2, MMP-9, and VEGF levels in lung and liver metastasis tissues
(Figure 5E), indicating that NRF2 activation can promote BC metastasis by increasing metastasis-associated genes. Together, our results strongly suggest that NRF2 activation plays an oncogenic role in DPP-4i-driven BC metastasis. 
A

4T1 cells treated with

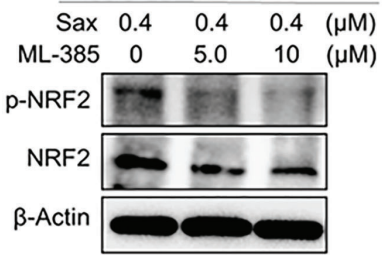

4T1 cells treated with

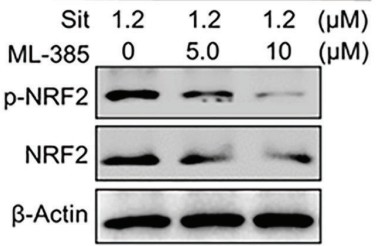

C
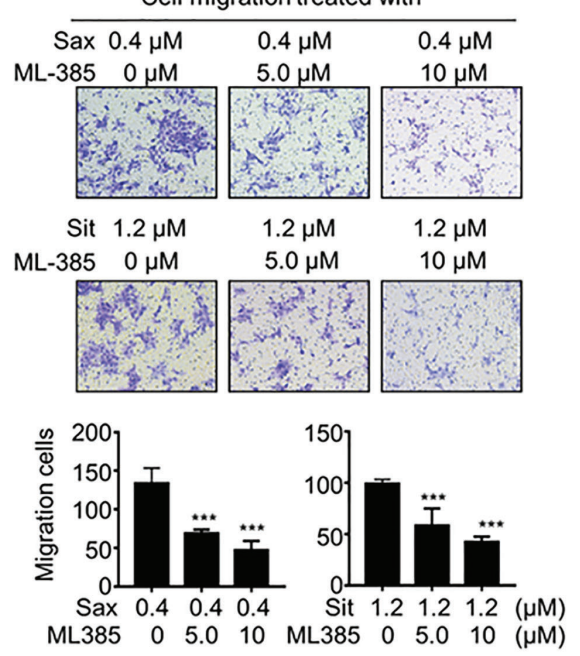

E

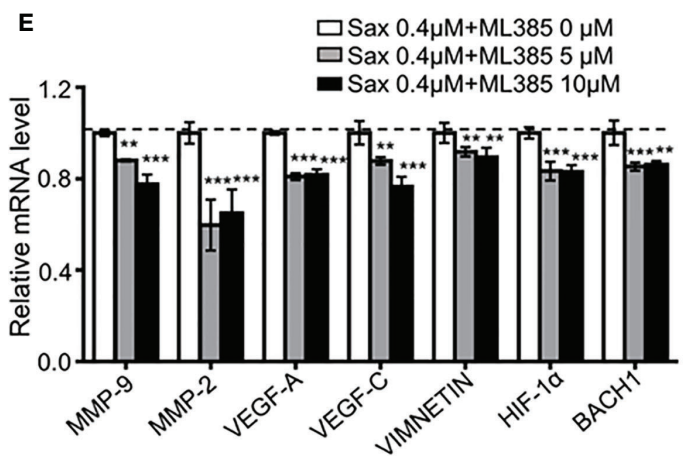

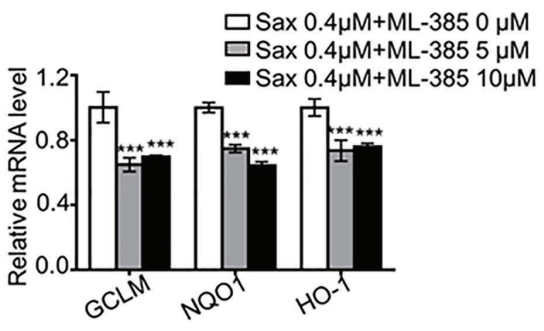

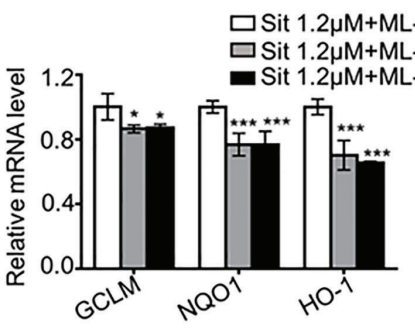

D
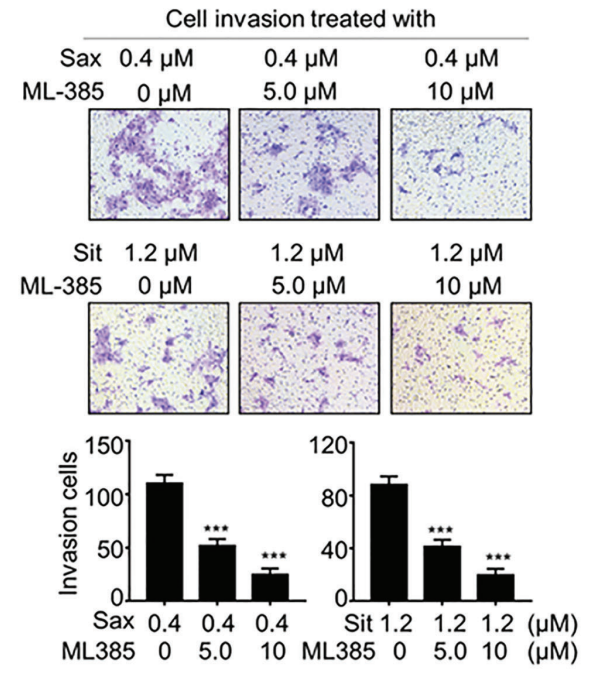

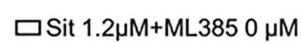

口Sit $1.2 \mu \mathrm{M}+\mathrm{ML} 3855 \mu \mathrm{M}$

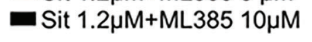

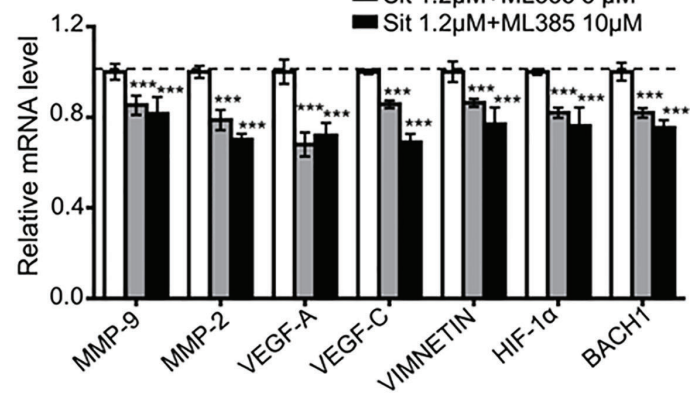

FIGURE 4 | Blockage NRF2 reverses DPP-4i-driven metastasis in vitro (A, B) NRF2 inhibition reverses DPP-4i-driven NRF2 activation. 4T1 cells were co-treated with DPP-4i and NRF2 specific inhibitor ML-385 for 4h. NRF2 and p-NRF2 expressions were detected by Western blotting (A) and NRF2-responsive genes levels were detected by qRT-PCR (B). $\beta$-actin gene was as an internal control. (C, D) NRF2 blockage attenuates DPP-4i-driven cell migration and invasion in vitro. 4T1 cells were subject to cell migration (C) and cell invasion (D) upon co-treatment with DPP-4i and ML-385 for 24 h. Migration or invasion cells were counted in 5 to 10 random fields. (E) NRF2 blockage abrogates DPP-4i-driven metastasis-associated gene levels. 4T1 cells were co-treated with or without DPP-4i and ML-385 and metastasis-associated gene levels were detected by qRT-PCR. $\beta$-actin gene was as an internal control. Data are presented as mean \pm SD of three independent experiments. Scale bar: $50 \mu \mathrm{m}$. Representative images are shown. ${ }^{*} p<0.05,{ }^{* \star} p<0.01$, and ${ }^{* \star *} p<0.001$ between the indicated groups determined by one-way analysis of variance (ANOVA). 
A

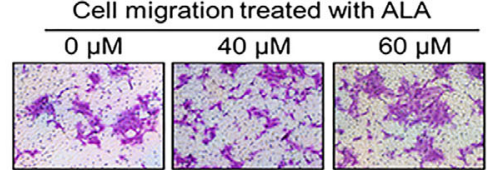

Cell invasion treated with ALA
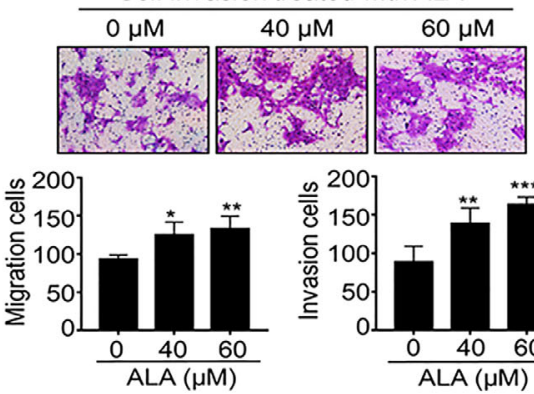

C
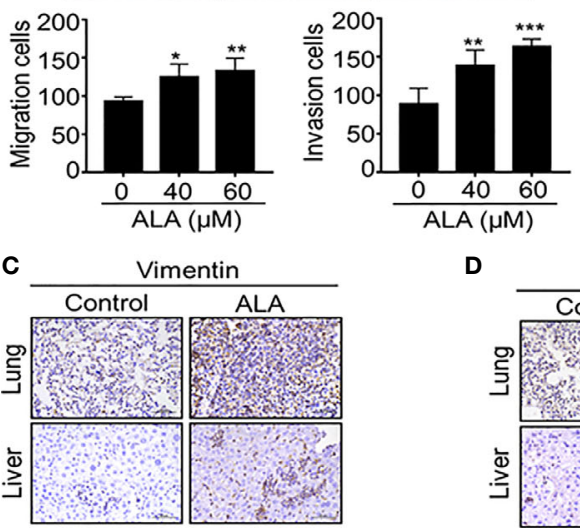

D
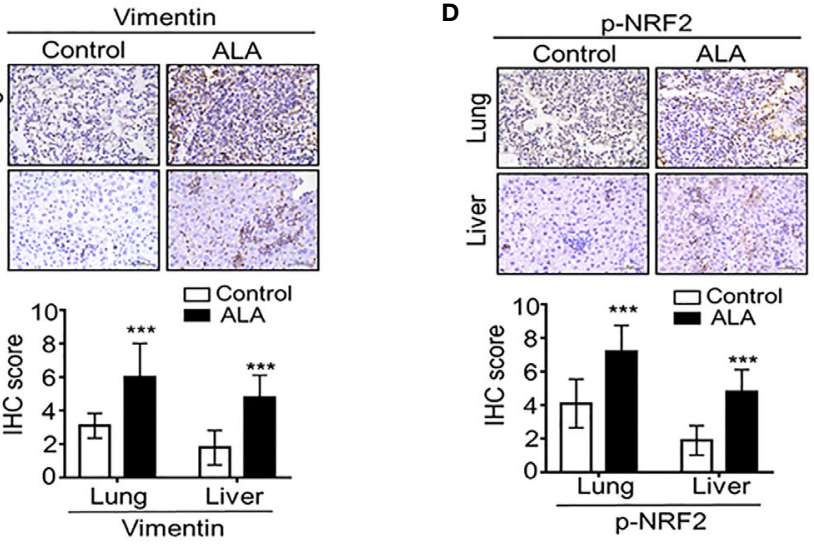

E
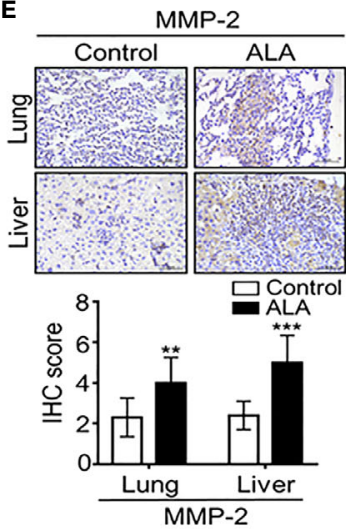
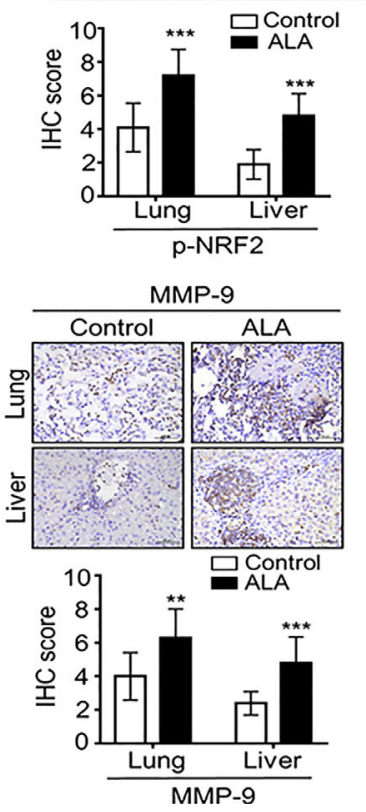

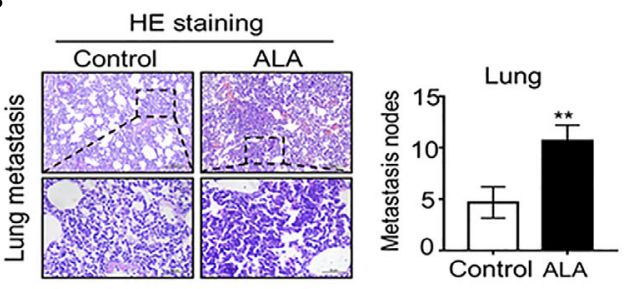

HE staining

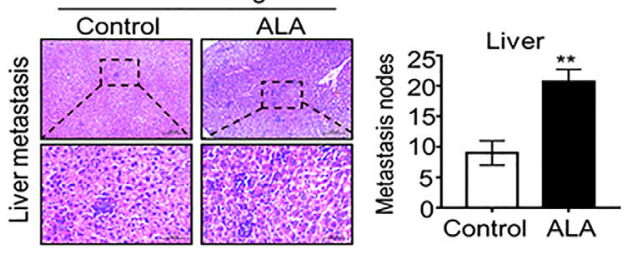

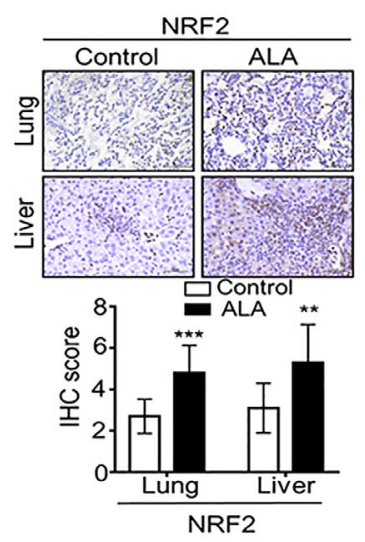

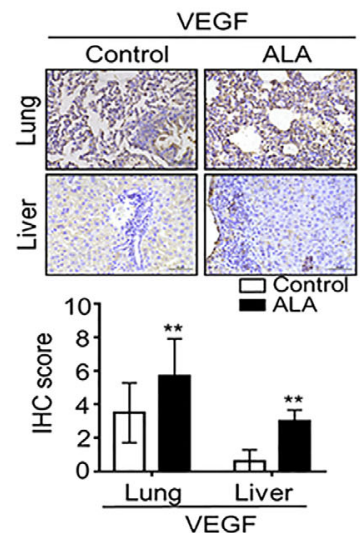

FIGURE 5 | Pharmaceutical NRF2 activation promotes BC metastasis in vitro and in vitro. (A) NRF2 activator ALA promotes BC cell migration and cell invasion. 4T1 cells were subject to cell migration and cell invasion assay upon ALA treatment. Migration or invasion cells were counted in 5 to 10 random fields. (B, C) ALA accelerates BC lung and liver metastasis in vivo. $1.0 \times 10^{5} 4 \mathrm{~T} 1$ cells in $100 \mu \mathrm{l}$ PBS buffer were injected into in the left mammary fat pad of female NOD-SCID mice. Post-injection 3 to 5 days, experimental mice were peritoneally injected (i.p.) with or without ALA (80mg/kg) three times per week as described in M\&M. After the indicated time point, lungs and livers were collected and metastatic nodes were counted by H\&E staining (B). Micro-metastasis marker vimentin was detected by IHC staining (C). (D) ALA induces NRF2 activation in vivo. 4T1-bearing mice were treated with ALA as above mentioned and p-NRF2 and NRF2 were detected by IHC staining in metastatic lung and liver tissues. (E) ALA enhances metastasis-associated gene expression in vivo. 4T1-bearing mice were treated with ALA as above mentioned and metastasis-associated proteins MMP-9, MMP-2 and VEGF were detected by IHC staining in lung and liver metastatic nodes. Data are presented as mean \pm SD of three independent experiments. Scale bar: $50 \mu \mathrm{m}$. Representative images are shown. ${ }^{\star} \mathrm{p}<0.05,{ }^{{ }^{*}} \mathrm{p}<0.01$ and ${ }^{{ }^{* \star}} \mathrm{p}<0.001$ between the indicated groups determined by unpaired Student's $t$-test or the one-way analysis of variance (ANOVA). 


\section{Heme Oxygenase 1 (HO-1) Is a Critical Mediator of Oncogenic ROS-NRF2 Axis in DPP-4i-Driven BC Metastasis}

Given the oncogenic role of ROS-NRF2 activations in DPP-4idriven $\mathrm{BC}$ metastasis, we sought to explore the underlying mechanism of how ROS-NRF2 activation promotes BC metastasis. Recent studies reported aberrant $\mathrm{HO}-1$ activation in human cancers (18-22), promoting us to focus on the role of NRF2-responsive HO-1 in DPP-4i-driven BC metastasis. Firstly, we investigated whether DPP-4i could increase HO- 1 activation via ROS-NRF2 axis-dependent manner. Using Western blot and IHC analysis, we observed that DPP-4i significantly increased HO-1 expression in vitro and in vivo (Figures $\mathbf{6 A}, \mathbf{B}$ ). In addition, we also found that DPP-4i-driven HO-1 levels were significantly abrogated upon NAC treatment or ML-385 intervention (Figures 6C, D and Supplementary Figure S3). These data indicate that DPP-4i-driven HO-1 activation is ROSNRF2 axis-dependent in BC cells.

To further define the critical role of HO- 1 in DPP-4i-driven BC metastasis, using HO-1-IN-1 hydrochloride, a specific HO-1 inhibitor (12), we found that $\mathrm{HO}-1$ inhibition significantly decreased DPP-4i-driven BC cell migration and invasion (Figures 6E, F). Furthermore, we observed that DPP-4i-driven metastasis-associated gene levels were significantly attenuated after HO-1 inhibition in BC cells (Figure 6G), suggesting that HO-1 inhibition could reverse DPP-4i-driven BC metastasis. Overall, these results suggest that $\mathrm{HO}-1$ plays a critical role in oncogenic ROS-NRF2 axis-driven BC metastasis.

\section{HO-1 Promotes BC Metastasis by Activating NRF2 via a Positive-Feedback Loop}

Given the oncogenic role of NRF2 and HO-1 in DPP-4i -driven $\mathrm{BC}$ metastasis, we further defined the possible mechanism underlying how NRF2-responsive $\mathrm{HO}-1$ activation promotes $\mathrm{BC}$ metastasis. In $\mathrm{BC}$ cells, we observed that the HO-1 inhibitor not only decreased HO-1 expression but also significantly attenuated the DPP-4i-driven NRF2 and p-NRF2 expression (Figure 7A). Moreover, DPP-4i-driven NRF2/ARE transcriptional activation (Figure $7 \mathbf{B}$ ) and NRF2-responsive genes were also markedly abrogated by HO-1 inhibition (Figure 7C), indicating that HO-1 may feedback regulate NRF2 activation in DPP-4i-treated BC cells.

To investigate whether $\mathrm{HO}-1$ inhibition could attenuate NRF2-driven BC metastasis, we conducted a dual pharmacological intervention assay using $\mathrm{HO}-1$ inhibitor and NRF2 activator in BC cells. As shown in Figures 7D, E, ALA treatment markedly enhanced NRF2, p-NRF2 and HO-1 expressions, whereas HO-1 inhibition significantly abrogated ALA-induced HO-1, NRF2 and p-NRF2 expressions. Meanwhile, ALA-induced NRF2/ARE transcriptional activation was also attenuated by $\mathrm{HO}-1$ inhibition (Figure 7F), indicating a positive-feedback regulation between $\mathrm{HO}-1$ and NRF2. Furthermore, we observed that ALA-driven cell migration and invasion were significantly reversed by $\mathrm{HO}-1$ inhibitor in $\mathrm{BC}$ cells
(Figure 7G), while ALA-induced NRF2 downstream targets as well as metastasis-associated genes levels were markedly inhibited by HO-1 inhibition (Figures $7 \mathbf{H}, \mathbf{I}$ and Supplementary Figure S2B), suggesting HO-1 inhibition can directly antagonize NRF2-driven BC metastasis in vitro. Thus, these results suggest that $\mathrm{HO}-1$ activation contributes to oncogenic NRF2 activation via a positivefeedback loop, promoting a serial of metastasis-associated gene expressions, thereby leading to $\mathrm{BC}$ metastasis.

\section{DISCUSSION}

Elucidating the potential side effect of antidiabetic agents in BC metastasis may shed new light on minimizing the risk of DPP-4i in diabetic patients with BC. 4T1 cells originally isolated from $\mathrm{BALB} / \mathrm{c}$ mice share many characteristics with naturally occurring human breast cancer and metastasize to distant organs via the hematogenous route, making it as an ideal model for mimicking the metastatic and advanced stages of human breast cancer (11). In our efforts to understand the effect of antidiabetic agents in existing $\mathrm{BC}$, we utilized severe combined immunodeficient NOD-SCID mice to establish a spontaneous BC metastasis mice model to better understand the effect of DPP-4i on biological behaviors of BC cells itself under immune-deficient status. Here, we revealed that DPP-4i promoted BC metastasis in vitro and in vivo, which can be abrogated by the pharmacological inhibition of ROS, NRF2 and HO-1 respectively. Mechanistically, DPP-4i induced excessive oxidative stress by triggering ROS production, resulting in oncogenic NRF2 and HO-1 activations. Moreover, HO-1 activation promoted oncogenic NRF2 activation via a positive feedback loop, promoting a series of metastasisassociated genes expression. Our study provides a critical profile of DPP-4i and aberrant NRF2 activation in BC metastasis.

Our finding demonstrates that DPP-4i promotes $\mathrm{BC}$ metastasis by triggering oxidative stress via ROS generation, improving our understanding of the role of oxidative stress in $\mathrm{BC}$ progression. Our previous finding suggested that DPP-4i promoted human BC metastasis through NRF2-mediated antioxidative stress (6). Here, our data clearly showed DPP-4i promoted $\mathrm{BC}$ metastasis by triggering aberrant oxidative stress via ROS overproduction while ROS scavenger significantly attenuated DPP-4i-induced BC metastasis in vitro. However, recent contradicting results have raised a possibility that ROS limits distant metastasis $(6,18)$. There is a possibility that specific characteristics of antioxidants and cell lines with different gene mutations may contribute to the contradictory results regarding the role of ROS and antioxidant regents in tumor metastasis (6). Our results strongly suggest that ROS-mediated oxidative stress plays an essential role in DPP-4i-driven BC metastasis, further improving our understanding of the role of DPP-4i in the BC progression.

Our study also reveals an oncogenic NRF2 activation in DPP$4 \mathrm{i}$-induced $\mathrm{BC}$ metastasis, improving our understanding of the side-effect of DPP-4i in tumor progression. NRF2 has long been identified as a well-known anti-cancer molecular while a serial of 


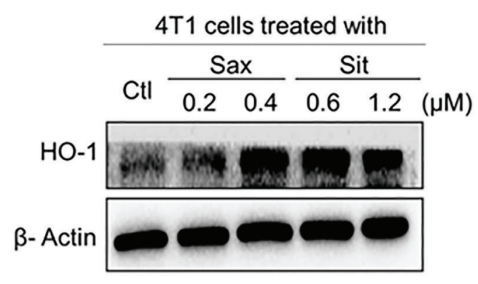

C
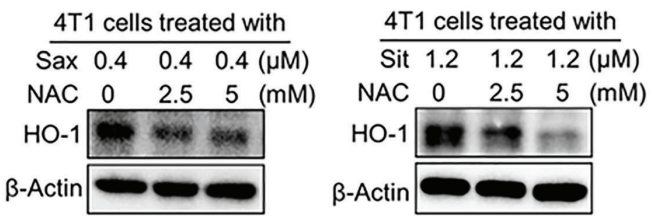

E
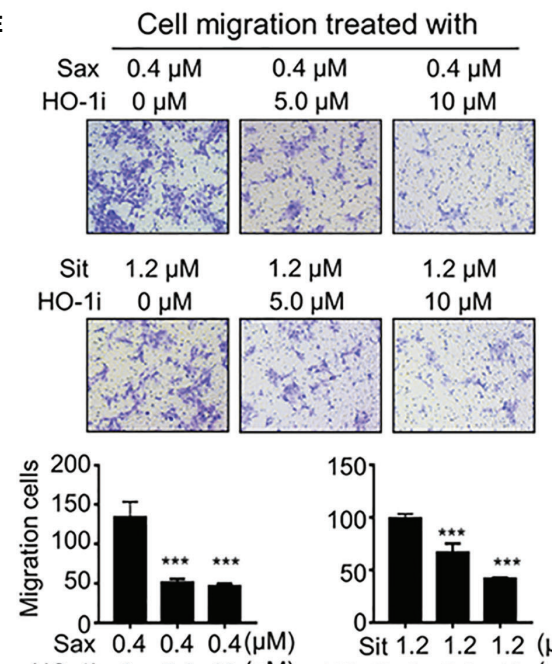

$\begin{array}{llllllll}\mathrm{HO}-1 \mathrm{i} & 0 & 5.0 & 10(\mu \mathrm{M}) & \mathrm{HO}-1 \mathrm{i} & 0 & 5.0 & 10\end{array}$

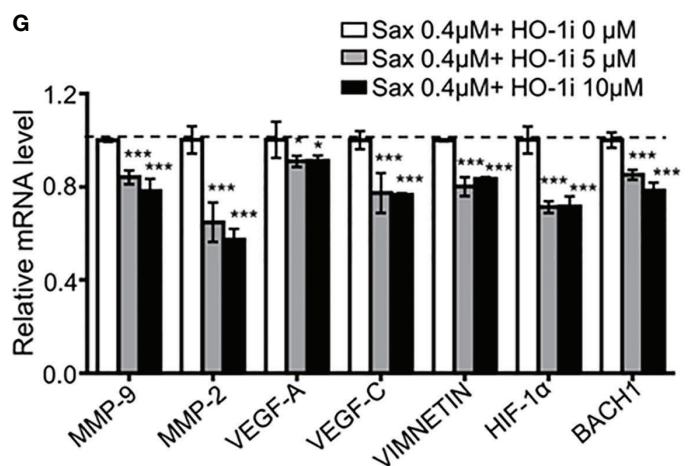

B
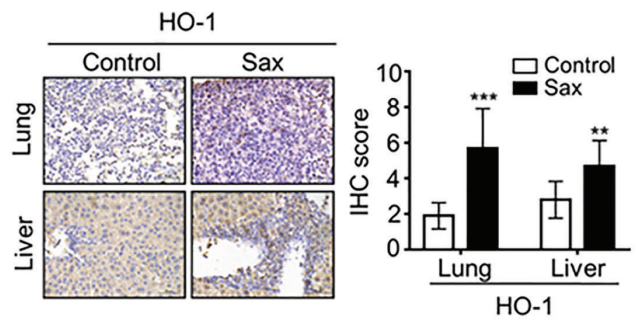

D

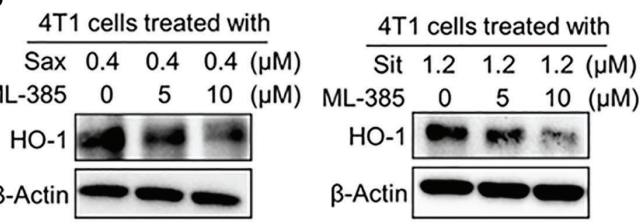

$\mathbf{F}$
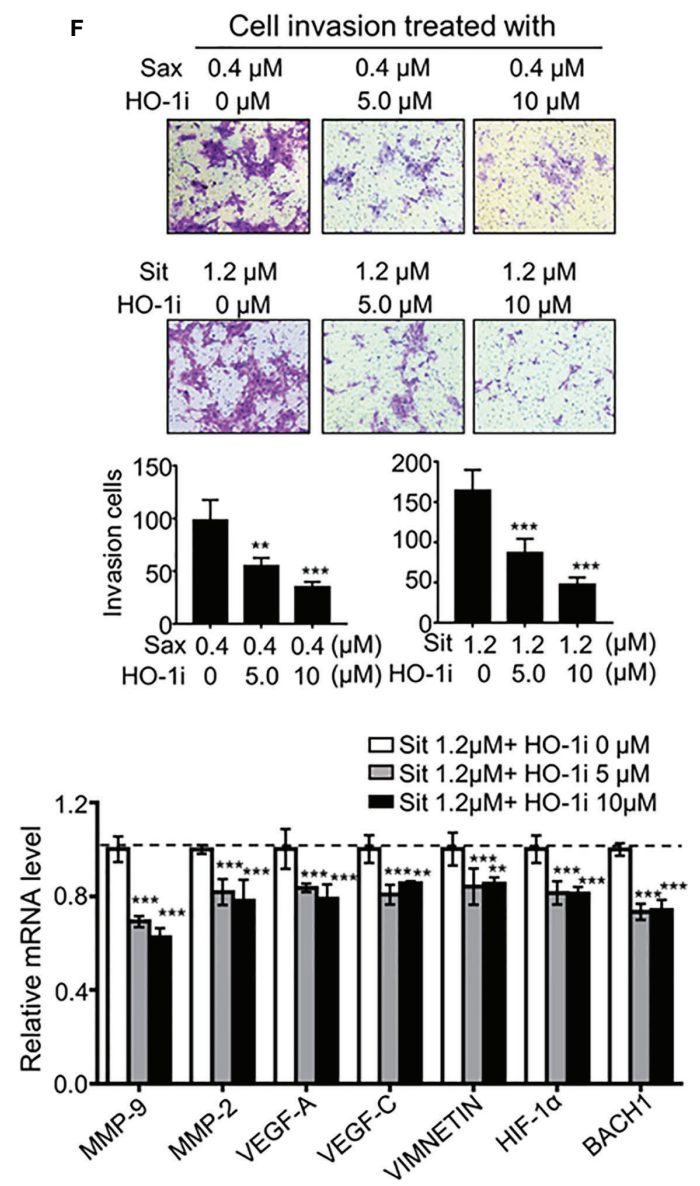

FIGURE 6 | ROS-NRF2 dependent HO-1 activation is critical to DPP-4i-driven BC metastasis. (A, B) DPP-4i enhances NRF2-responsive HO-1 activation. HO-1 expression was analyzed by western blotting in DPP-4i-treated 4T1 cells (A) and by IHC staining in lung and liver metastasis tissues (B), respectively. (C) ROS inhibition abrogates DPP4i-induced HO-1 activation. 4T1 cells were co-treated with DPP-4i and NAC and $\mathrm{HO}-1$ expression were detected by western blotting. $\beta$ actin was a loading control. (D) NRF2 inhibition attenuates DPP-4i-induced HO-1 activation. 4T1 cells were co-treated with DPP-4i and NRF2 specific inhibitor ML385, and $\mathrm{HO}-1$ expression were detected by western blotting. $\beta$-actin was a loading control. (E, F) HO-1 blockage reverses DPP-4i -driven BC cell migration and cell invasion in vitro. 4T1 cells were subject to cell migration (E) and cell invasion (F) upon co-treatment with DPP-4i and HO-1 specific inhibitor for 24 h. Migration or invasion cells were counted in 5 to 10 random fields. (G) HO-1 blockage abrogates DPP-4i-driven metastasis-associated gene levels. $4 \mathrm{~T} 1$ cells were co-treated with or without DPP-4i and HO-1 inhibitor for $4 \mathrm{~h}$ and metastasis-associated gene levels were detected by qRT-PCR. $\beta$-Actin gene was as an internal control. Data are presented as mean \pm SD of three independent experiments. Representative images are shown. Scale bar: $50 \mu \mathrm{m}$. ${ }^{*} \mathrm{p}<0.05$, ${ }^{* *} \mathrm{p}<0.01$, and ${ }^{* \star \star} \mathrm{p}<0.001$ between the indicated groups determined by one-way analysis of variance (ANOVA). 
A

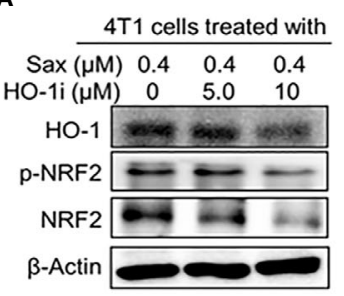

B

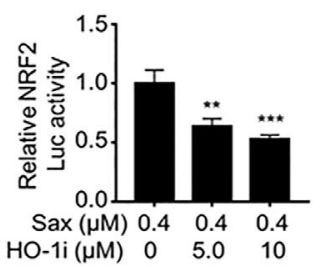

D

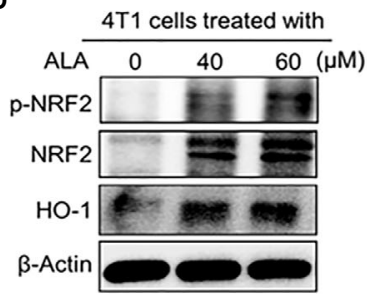

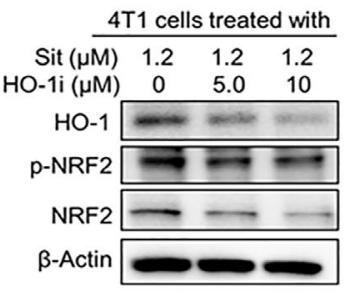
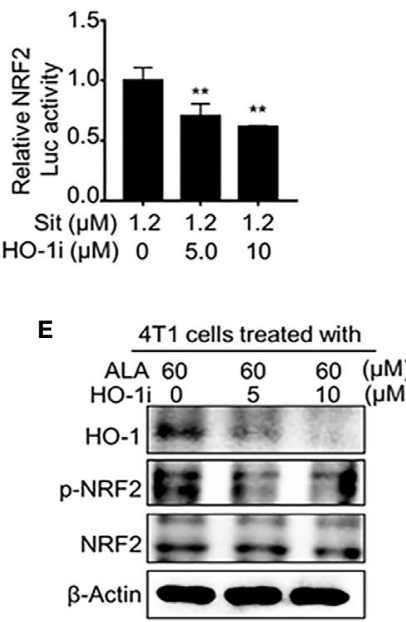
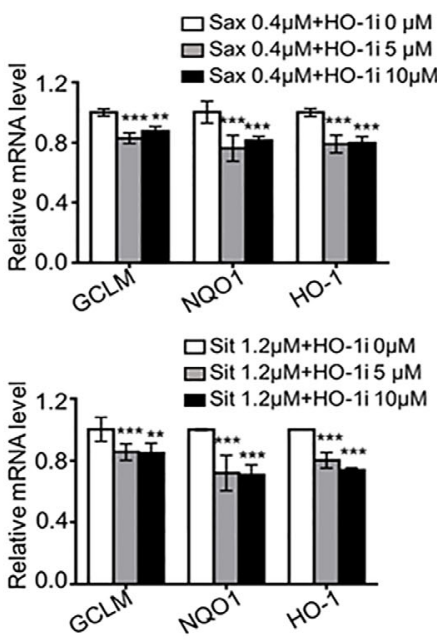

$\mathbf{F}$

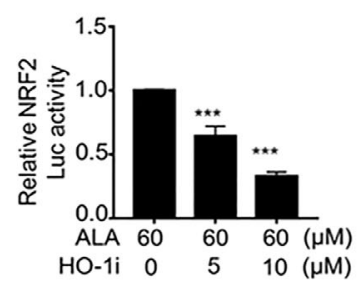

G
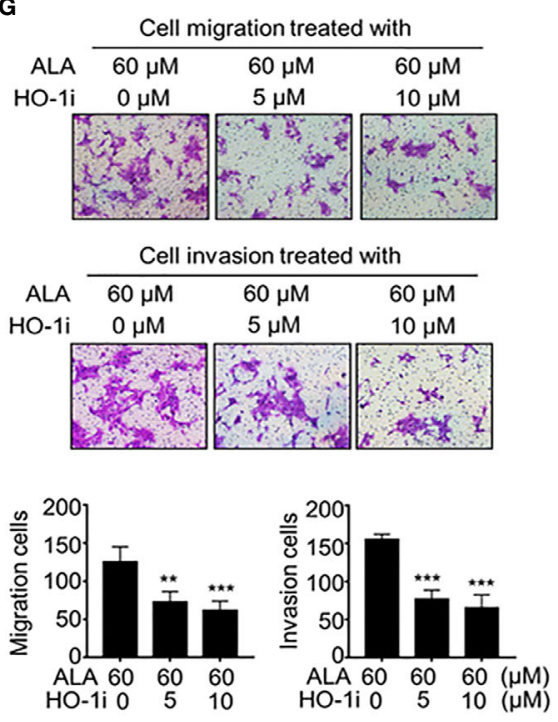
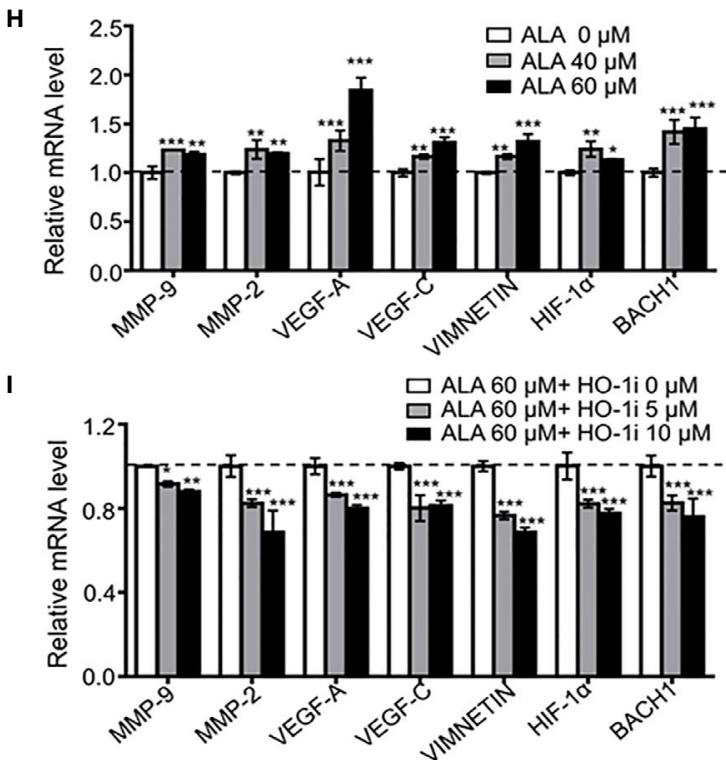

FIGURE 7 | HO-1 reinforces NRF2 activation via a positive feedback loop. (A-C) HO-1 blockage abrogates DPP-4i-driven NRF2 activation in vitro. 4T1 cells were co-treatment with DPP-4i and HO-1 specific inhibitor (HO-1i). HO-1, p-NRF2, and NRF2 expressions were detected by western blotting (A) and NRF2/ARE transcriptional activation was analyzed by luciferase reporter gene assay (B). NRF2-responsive genes were detected by qRT-PCR (C). $\beta$-Actin gene was as a control. (D) NRF2 activator increases NRF2 activation in 4T1 cells. p-NRF2, NRF2, and HO-1 expressions in ALA-treated 4T1 cells were detected by Western blotting. $\beta$-actin was as a loading control. (E, F) HO-1 blockage abrogates ALA-induced NRF2 activation. 4T1 cells were co-treated with or without ALA and HO-1 inhibitor. HO-1, p-NRF2 and NRF2 expressions were analyzed by Western blotting (E). NRF2/ARE transcriptional activation was detected by luciferase reporter gene assay (F). (G) HO-1 inhibition attenuates ALA-induced BC migration and cell invasion. 4T1 cells were co-treated with ALA and HO-1 inhibitor. Cell migration and cell invasion assays were performed and migration or invasion cells were counted in 5 to 10 random fields. (H, I) HO-1 blockage abrogates ALA-driven metastasisassociated gene levels. 4T1 cells were treated with ALA alone $\mathbf{( H )}$ or were co-treated with ALA and HO-1 inhibitor for 4h (I). Metastasis-associated gene levels were detected by qRT-PCR. $\beta$-actin gene was as an internal control. Representative images are shown. Data are presented as mean \pm SD of three independent experiments. Scale bar: $50 \mu \mathrm{m}$. ${ }^{*} \mathrm{p}<0.05,{ }^{* *} \mathrm{p}<0.01$, and ${ }^{* * *} \mathrm{p}<0.001$ between the indicated groups determined by one-way analysis of variance (ANOVA). 
antioxidants suppresses tumor progression (19, 20). However, recent evidences suggest the oncogenic profile of NRF2 activation in tumor progression and show that aberrant NRF2 accelerates cancer progression while antioxidants accelerate migration and invasion of cancer cells $(6,21,22)$. Our recent report also showed that mitochondrial GRIM-19 deficiency accelerated human gastric cancer metastasis through the oncogenic ROS-NRF2-HO-1 axis via a positive-feedback NRF2-HO-1 loop. Therefore, this study not only offers novel insights to the oncogenic role of NRF2 in BC progression, but also provides new strategies to alleviate the dark side of NRF2 by targeting HO-1. Here, our data demonstrated that DPP-4i promoted $\mathrm{BC}$ metastasis by ROS-dependent NRF2 activation while specific inhibition of ROS or NRF2 abrogated DPP-4idriven $\mathrm{BC}$ metastasis, which is consistent with recent findings that NRF2 inhibitors could antagonize human cancers (23-25). Therefore, our results strongly suggest that NRF2 plays an oncogenic role in DPP-4i-driven BC metastasis.

Our finding reveals that NRF2-responsive HO-1 contributes to DPP-4i-induced oncogenic NRF2 activation via a positive-feedback NRF2-HO-1 loop, not only providing novel mechanism insights into the oncogenic NRF2 in BC metastasis, but also offering new strategies to inhibit the dark side of oncogenic NRF2 activation by targeting its downstream target $\mathrm{HO}-1$. Our results demonstrate that NAC suppresses DPP-4i-driven BC metastasis whereas NRF2 activator ALA accelerates $\mathrm{BC}$ metastasis, raising a topic concerning the paradox profile of antioxidants in cancer progression. HO-1, as one of NRF2 downstream targets, plays a critical role in the maintenance of cellular redox homeostasis (12, 21). However, recent evidences also showed that aberrant HO-1 in human cancers contributes to cancer metastasis (12, 26-30). Here, our results showed that the pharmacological ROS or NRF2 inhibition decreased HO-1 level while $\mathrm{HO}-1$ inhibition abrogated DPP-4i-driven BC metastasis, suggesting that NRF2-responsive HO-1 activation is required to DPP-4i-driven BC metastasis. Moreover, recent finding also showed that HO-1 activation was correlated with metastasis-associated genes such as MMP-9, VEGFA, HIF-1 $\alpha$, and BACH-1 (26-28, 31-33). Our data also showed that HO-1 inhibition blocked DPP-4i-driven metastasis-associated genes in BC cells. Our recent report also showed that oncogenic ROSNRF2-HO-1 axis contributes to mitochondrial GRIM-19 deficiency-driven metastasis in human gastric cancer via a positive-feedback NRF2-HO-1 loop (12). Thus, HO-1, as a critical downstream target of the ROS-NRF2 axis, feedback promotes oncogenic NRF2 activation, resulting in upregulation of metastasis-associated genes, thereby facilitating $\mathrm{BC}$ metastasis. Our finding provides a reasonable explanation for the NRF2driven $\mathrm{BC}$ metastasis, and suggests that more comprehensive preclinical and clinical studies should be performed to ensure the safety of antioxidants in cancer patients.

In summary, our data suggest that DPP-4i accelerates murine BC metastasis through the oncogenic ROS-NRF2-HO-1 axis via a positive-feedback NRF2-HO-1 loop. This finding not only offers a mechanistic insight to DPP-4i-driven BC metastasis by ROS-NRF2 activation, but also provides new avenues to eliminate the "dark side" of NRF2 by targeting HO-1.

\section{DATA AVAILABILITY STATEMENT}

The raw data supporting the conclusions of this article will be made available by the authors, without undue reservation.

\section{ETHICS STATEMENT}

The animal study was reviewed and approved by the Institutional Animal Care and Use Committee of Children's Hospital of Chongqing Medical University.

\section{AUTHOR CONTRIBUTIONS}

Conception and design: RL, XZ, MY, YH. Development of methodology: RL, XZ, XW, BX, XX. Acquisition of data (provided animals, provided facilities, etc.): RL, XZ, XW, BX, XX. Analysis and interpretation of data (e.g., statistical analysis): RL, XZ, JF, BX, YH. Writing, review, and/or revision of the manuscript: RL, $\mathrm{XZ}, \mathrm{MY}, \mathrm{LB}, \mathrm{YH}$. Administrative, technical, or material support (i.e., reporting or organizing data, constructing databases): $\mathrm{RL}, \mathrm{XX}, \mathrm{JF}$, XW, BX. Study supervision: YH. All authors contributed to the article and approved the submitted version.

\section{FUNDING}

This study was partly supported by Chongqing basic and frontier research project (CSTC2018jcyjAX0218) and Chongqing Yuzhong District Sci \& Tech Research Project (20190106).

\section{SUPPLEMENTARY MATERIAL}

The Supplementary Material for this article can be found online at: https://www.frontiersin.org/articles/10.3389/fonc.2021.679816/ full\#supplementary-material

\footnotetext{
Supplementary Figure 1 | DPP-4i induces aberrant oxidative stress in BC cells. Oxidative stress status in DPP-4i-treated BC cells was evaluated by analyzing ATP content (A), NADP+/NADPH ratio (B) and GSH/GSSG ratio (C). Data are presented as mean \pm SD of three independent experiments. ${ }^{*} p<0.05$, ${ }^{* *} p<0.01$, and ${ }^{* \star *} \mathrm{p}<0.001$ between the indicated groups determined by one-way analysis of variance (ANOVA)
}

Supplementary Figure 2 | HO-1 blockage abrogates ALA-induced NRF2responsive genes expression. 4T1 cells were treated with ALA (A) or were cotreated with ALA and HO-1 inhibitor (B). The expression of NRF2-responsive genes was analyzed by Real-time PCR. $\beta$-actin was as an internal control. Data are presented as mean \pm SD of three independent experiments. ${ }^{*} p<0.05$, ${ }^{* *} p<0.01$, and ${ }^{* * *} \mathrm{p}<0.001$ between the indicated groups determined by one-way analysis of variance (ANOVA).

Supplementary Figure 3 | Relative expression analysis of $\mathrm{HO}-1$ protein after cotreatment of DPP-4i with NAC or ML-385. 4T1 cells were co-treated with DPP-4i and NAC (A) or ML-385 (B). HO-1 expression was detected by western blotting as shown in Figure 6C and 6D. Relative $\mathrm{HO}-1$ expression was obtained by analyzing the ratio of $\mathrm{HO}-1$ and $\beta$-Actin. Data are presented as mean $\pm \mathrm{SD}$ of three independent experiments. ${ }^{\star \star} p<0.01$ and ${ }^{* \star *} p<0.001$ between the indicated groups determined by one-way analysis of variance (ANOVA). 


\section{REFERENCES}

1. Giovannucci E, Harlan DM, Archer MC, Bergenstal RM, Gapstur SM, Habel LA, et al. Diabetes and Cancer: A Consensus Report. CA Cancer J Clin (2010) 60:207-21. doi: 10.3322/caac.20078

2. Walker JJ, Johnson JA, Wild SH. Diabetes Treatments and Cancer Risk: The Importance of Considering Aspects of Drug Exposure. Lancet Diabetes Endocrinol (2013) 1:132-9. doi: 10.1016/S2213-8587(13)70028-7

3. Lee M, Sun J, Han M, Cho Y, Lee JY, Nam CM, et al. Nationwide Trends in Pancreatitis and Pancreatic Cancer Risk Among Patients With Newly Diagnosed Type 2 Diabetes Receiving Dipeptidyl Peptidase 4 Inhibitors. Diabetes Care (2019) 42:2057-64. doi: 10.2337/dc18-2195

4. Kawaguchi T, Nakano D, Koga H, Torimura T. Effects of a DPP4 Inhibitor on Progression of NASH-related HCC and the P62/ Keap1/Nrf2-Pentose Phosphate Pathway in a Mouse Model. Liver Cancer (2019) 8:359-72. doi: $10.1159 / 000491763$

5. Barreira da Silva R, Laird ME, Yatim N, Fiette L, Ingersoll MA, Albert ML. Dipeptidylpeptidase 4 Inhibition Enhances Lymphocyte Trafficking, Improving Both Naturally Occurring Tumor Immunity and Immunotherapy. Nat Immunol (2015) 16:850-8. doi: 10.1038/ni.3201

6. Wang H, Liu X, Long M, Huang Y, Zhang L, Zhang R, et al. Nrf2 Activation by Antioxidant Antidiabetic Agents Accelerates Tumor Metastasis. Sci Transl Med (2016) 8:334ra51. doi: 10.1126/scitranslmed.aad6095

7. Dunn GP, Old LJ, Schreiber RD. The Three Es of Cancer Immunoediting. Annu Rev Immunol (2004) 22:329-60. doi: 10.1146/annurev.immunol. 22.012703.104803

8. Dunn GP, Old LJ, Schreiber RD. The Immunobiology of Cancer Immunosurveillance and Immunoediting. Immunity (2004) 21:137-48. doi: 10.1016/j.immuni.2004.07.017

9. Schreiber RD, Old LJ, Smyth MJ. Cancer Immunoediting: Integrating Immunity's Roles in Cancer Suppression and Promotion. Science (2011) 331:1565-70. doi: 10.1126/science. 1203486

10. Bui JD, Schreiber RD. Cancer Immunosurveillance, Immunoediting and Inflammation: Independent or Interdependent Processes? Curr Opin Immunol (2007) 19:203-8. doi: 10.1016/j.coi.2007.02.001

11. Huang Y, Ma C, Zhang Q, Ye J, Wang F, Zhang Y, et al. CD4+ and CD8+ T Cells Have Opposing Roles in Breast Cancer Progression and Outcome. Oncotarget (2015) 6:17462-78. doi: 10.18632/oncotarget.3958

12. Wang X, Ye T, Xue B, Yang M, Li R, Xu X, et al. Mitochondrial GRIM-19 Deficiency Facilitates Gastric Cancer Metastasis Through Oncogenic ROSNRF2-HO-1 Axis Via a NRF2-HO-1 Loop. Gastric Cancer (2021) 24:117-32. doi: 10.1007/s10120-020-01111-2

13. Ye T, Yang M, Huang D, Wang X, Xue B, Tian N, et al. MicroRNA-7 as a Potential Therapeutic Target for Aberrant NF-Kappab-Driven Distant Metastasis of Gastric Cancer. J Exp Clin Cancer Res (2019) 38:55. doi: 10.1186/s13046-019-1074-6

14. Huang Y, Yang M, Yang H, Zeng Z. Upregulation of the GRIM-19 Gene Suppresses Invasion and Metastasis of Human Gastric Cancer Sgc-7901 Cell Line. Exp Cell Res (2010) 316:2061-70. doi: 10.1016/j.yexcr.2010.05.010

15. Huang Y, Yang M, Hu H, Zhao X, Bao L, Huang D, et al. Mitochondrial GRIM-19 as a Potential Therapeutic Target for STAT3-dependent Carcinogenesis of Gastric Cancer. Oncotarget (2016) 7:41404-20. doi: 10.18632/oncotarget.9167

16. Liu RH, Yang MH, Xiang H, Bao LM, Yang HA, Yue LW, et al. Depletion of OLFM4 Gene Inhibits Cell Growth and Increases Sensitization to Hydrogen Peroxide and Tumor Necrosis Factor-Alpha Induced-Apoptosis in Gastric Cancer Cells. J BioMed Sci (2012) 19:38. doi: 10.1186/1423-0127-19-38

17. Xue B, Li Y, Wang X, Li R, Zeng X, Yang M, et al. Taqman-MGB Probe Quantitative PCR Assays to Genotype and Quantify Three Mtdna Mutations of Leber Hereditary Optic Neuropathy. Sci Rep (2020) 10:12264. doi: 10.1038/ s41598-020-69220-7
18. Piskounova E, Agathocleous M, Murphy MM, Hu Z, Huddlestun SE, Zhao Z, et al. Oxidative Stress Inhibits Distant Metastasis by Human Melanoma Cells. Nature (2015) 527:186-91. doi: 10.1038/nature15726

19. de la Vega MR, Chapman E, Zhang DD. NRF2 and the Hallmarks of Cancer. Cancer Cell (2018) 34:21-43. doi: 10.1016/j.ccell.2018.03.022

20. Sporn MB, Liby KT. NRF2 and Cancer: The Good, the Bad and the Importance of Context. Nat Rev Cancer (2012) 12:564-71. doi: 10.1038/ nrc3278

21. Le Gal K, Ibrahim MX, Wiel C, Sayin VI, Akula MK, Karlsson C, et al. Antioxidants can Increase Melanoma Metastasis in Mice. Sci Transl Med (2015) 7:308re8. doi: 10.1126/scitranslmed.aad3740

22. DeNicola GM, Karreth FA, Humpton TJ, Gopinathan A, Wei C, Frese K, et al. Oncogene-Induced Nrf2 Transcription Promotes Ros Detoxification and Tumorigenesis. Nature (2011) 475:106-9. doi: 10.1038/nature10189

23. Lau A, Villeneuve NF, Sun Z, Wong PK, Zhang DD. Dual Roles of Nrf2 in Cancer. Pharmacol Res (2008) 58:262-70. doi: 10.1016/j.phrs.2008.09.003

24. Hayes JD, McMahon M. NRF2 and KEAP1 Mutations: Permanent Activation of an Adaptive Response in Cancer. Trends Biochem Sci (2009) 34:176-88. doi: 10.1016/j.tibs.2008.12.008

25. Singh A, Venkannagari S, Oh KH, Zhang YQ, Rohde JM, Liu L, et al. Small Molecule Inhibitor of NRF2 Selectively Intervenes Therapeutic Resistance in KEAP1-Deficient Nsclc Tumors. ACS Chem Biol (2016) 11:3214-25. doi: 10.1021/acschembio.6b00651

26. Ryter SW, Choi AM. Heme Oxygenase-1: Redox Regulation of a Stress Protein in Lung and Cell Culture Models. Antioxid Redox Signal (2005) 7:80-91. doi: 10.1089 /ars.2005.7.80

27. Tsai JR, Wang HM, Liu PL, Chen YH, Yang MC, Chou SH, et al. High Expression of Heme Oxygenase-1 is Associated With Tumor Invasiveness and Poor Clinical Outcome in non-Small Cell Lung Cancer Patients. Cell Oncol (Dordr) (2012) 35:461-71. doi: 10.1007/s13402-012-0105-5

28. Prawan A, Kundu JK, Surh YJ. Molecular Basis of Heme Oxygenase-1 Induction: Implications for Chemoprevention and Chemoprotection. Antioxid Redox Signal (2005) 7:1688-703. doi: 10.1089/ars.2005.7.1688

29. Yin Y, Liu Q, Wang B, Chen G, Xu L, Zhou H. Expression and Function of Heme Oxygenase-1 in Human Gastric Cancer. Exp Biol Med (Maywood) (2012) 237:362-71. doi: 10.1258/ebm.2011.011193

30. Lu DY, Yeh WL, Huang SM, Tang CH, Lin HY, Chou SJ. Osteopontin Increases Heme Oxygenase-1 Expression and Subsequently Induces Cell Migration and Invasion in Glioma Cells. Neuro Oncol (2012) 14:1367-78. doi: 10.1093/neuonc/nos262

31. Wiel C, Le Gal K, Ibrahim MX, Jahangir CA, Kashif M, Yao H, et al. Bach1 Stabilization by Antioxidants Stimulates Lung Cancer Metastasis. Cell (2019) 178:330-45 e22. doi: 10.1016/j.cell.2019.06.005

32. Anderson NM, Simon MC. Bach1 Orchestrates Lung Cancer Metastasis. Cell (2019) 178:265-7. doi: 10.1016/j.cell.2019.06.020

33. Lignitto L, LeBoeuf SE, Homer H, Jiang S, Askenazi M, Karakousi TR, et al. Nrf2 Activation Promotes Lung Cancer Metastasis by Inhibiting the Degradation of Bach1. Cell (2019) 178:316-29 e18. doi: 10.1016/ j.cell.2019.06.003

Conflict of Interest: The authors declare that the research was conducted in the absence of any commercial or financial relationships that could be construed as a potential conflict of interest.

Copyright $\odot 2021$ Li, Zeng, Yang, Xu, Feng, Bao, Xue, Wang and Huang. This is an open-access article distributed under the terms of the Creative Commons Attribution License (CC BY). The use, distribution or reproduction in other forums is permitted, provided the original author(s) and the copyright owner(s) are credited and that the original publication in this journal is cited, in accordance with accepted academic practice. No use, distribution or reproduction is permitted which does not comply with these terms. 